TRANSACTIONS OF THE

AMERICAN MATHEMATICAL SOCIETY

Volume 348, Number 6, June 1996

\title{
PRIME SPECTRA OF QUANTUM SEMISIMPLE GROUPS
}

\author{
K. A. BROWN AND K. R. GOODEARL
}

\begin{abstract}
We study the prime ideal spaces of the quantized function algebras $R_{q}[G]$, for $G$ a semisimple Lie group and $q$ an indeterminate. Our method is to examine the structure of algebras satisfying a set of seven hypotheses, and then to demonstrate, using work of Joseph, Hodges and Levasseur, that the algebras $R_{q}[G]$ satisfy this list of assumptions. Rings satisfying the assumptions are shown to satisfy normal separation, and therefore Jategaonkar's strong second layer condition. For such rings much representation-theoretic information is carried by the graph of links of the prime spectrum, and so we proceed to a detailed study of the prime links of algebras satisfying the list of assumptions. Homogeneity is a key feature - it is proved that the clique of any prime ideal coincides with its orbit under a finite rank free abelian group of automorphisms. Bounds on the ranks of these groups are obtained in the case of $R_{q}[G]$. In the final section the results are specialized to the case $G=S L_{n}(\mathbb{C})$, where detailed calculations can be used to illustrate the general results. As a preliminary set of examples we show also that the multiparameter quantum coordinate rings of affine $n$-space satisfy our axiom scheme when the group generated by the parameters is torsionfree.
\end{abstract}

\section{INTRODUCTION AND BACKGROUND}

0.1. Let $P$ and $Q$ be prime ideals of a noetherian $\operatorname{ring} R$. We say that $P$ is linked to $Q$, and write $P \rightsquigarrow Q$, if there is an ideal $A$ of $R$ with $P Q \subseteq A \subset P \cap Q$ such that $(P \cap Q) / A$ is a nonzero torsionfree left $(R / P)$-module and a torsionfree right $(R / Q)$-module. This condition can be simplified in case $R$ satisfies the second layer condition (see below). In that case, $P \rightsquigarrow Q$ if and only if $(P \cap Q) / P Q$ is faithful as a left $(R / P)$-module and as a right $(R / Q)$-module $[\mathbf{3 9} ; \mathbf{1 0}, 1.4]$.

The graph of links of $R$ is the directed graph whose vertices are the points of spec $R$ (the set of prime ideals of $R$ ), with a directed edge from $P$ to $Q$ if and only if $P \rightsquigarrow Q$. The primes in the connected component of the graph of links containing $P$ constitute the clique of $P$, denoted clique $(P)$. There is a close connection between the graph of links of $R$ and the representation theory of $R$ (cf. [20], [4]); this connection is particularly strong when $R$ satisfies the strong second layer condition, which requires that, whenever $U$ is a cyclic uniform $R$-module with the annihilator

Received by the editors November 4, 1994 and, in revised form, September 5, 1995.

1991 Mathematics Subject Classification. Primary 16D30, 16D60, 16P40, 17 B37.

The research of the second author was partially supported by a grant from the National Science Foundation (USA). Part of the work was carried out while he visited the University of Glasgow Mathematics Department during October 1993, supported by the Edinburgh and London Mathematical Societies. Work on a revised version of the paper was completed in summer 1995 during a visit by both authors to the Department of Mathematics of the University of Washington, whom both thank for its hospitality. The travel costs of the first author were in part covered by a grant from the Carnegie Trust for the Universities of Scotland.

(C)1996 American Mathematical Society 
of each of its nonzero submodules being a prime $P$, no nonzero finitely generated submodule of the injective hull of $U$ can have annihilator equal to a prime other than $P$. Details of these concepts can be found in $[\mathbf{1 3}, \mathbf{2 0}]$.

In particular, links between primitive ideals in a noetherian ring $R$ with the strong second layer condition test for the existence of extensions of simple modules. Namely, if $S$ and $T$ are simple left $R$-modules and there exists a non-split extension $0 \rightarrow S \rightarrow M \rightarrow T \rightarrow 0$, then either $\operatorname{ann}_{R} S=\operatorname{ann}_{R} M$ or $\operatorname{ann}_{R}(S) \rightsquigarrow \operatorname{ann}_{R} T[\mathbf{2 0}$, $9.1 .2 ; \mathbf{1 3}, 11.4 ; \mathbf{3 1}, 4.3 .10]$. (The cited results are all stated for right modules; interpreting them for left modules requires reversing the link arrows.) Conversely, the existence of links implies the existence of certain non-split extensions (e.g., [4, $3.5 ; 13,11.2]$ ), although not necessarily extensions of the given simple modules. The connection is tight, however, in case $R / \operatorname{ann}_{R} S$ and $R / \operatorname{ann}_{R} T$ are artinian (e.g., if $S$ and $T$ are finite dimensional over a central subfield of $R$ ). In this case, a non-split extension $0 \rightarrow S \rightarrow M \rightarrow T \rightarrow 0$ exists if and only if $\operatorname{ann}_{R} S \rightsquigarrow \operatorname{ann}_{R} T$.

0.2. Let $q$ be an indeterminate, and let $\mathbf{g}$ be a finite dimensional complex semisimple Lie algebra. Generators and relations for the quantized enveloping algebra $U_{q}(\mathrm{~g})$ over a field $\mathbb{K} \supseteq \mathbb{Q}(q)$ are given in $[\mathbf{2 3}, 3.2 .9,5.1 .1]$. Being a Hopf algebra, $U_{q}(\mathrm{~g})$ possesses a Hopf dual

$$
U_{q}(\mathbf{g})^{\star}=\left\{f \in \operatorname{Hom}_{\mathbb{K}}\left(U_{q}(\mathbf{g}), \mathbb{K}\right) \mid f=0 \text { on some ideal of finite codimension }\right\}
$$

which is an algebra by virtue of the comultiplication on $U_{q}(\mathbf{g})$. The nature of the finite dimensional representations of $U_{q}(\mathbf{g})\left[\mathbf{2 3}\right.$, Section 4.3] implies that $U_{q}(\mathbf{g})^{\star}$ is a skew group ring over a finite elementary abelian 2-group, with coefficient ring $R_{q}[G]$, the so-called quantized function algebra of $G$ (see $[\mathbf{2 3}, 9.1 .1]$ ), which is a sub-Hopf algebra of $U_{q}(\mathbf{g})^{\star}$ and which is the main object of study of this paper. In general, $G$ is just a symbol, but when $\mathbb{K}=\mathbb{C}$ it denotes the connected, simply connected semisimple algebraic group with Lie algebra $\mathbf{g}$. For $\mathbf{g}=\mathbf{s l}_{n}(\mathbb{C})$ generators and relations for $R_{q}[G]$ are given in $[\mathbf{1 5}, 1.4 .1]$ (see (6.1) below).

0.3. Let $A=R_{q}[G]$ be as in (0.2). The prime and primitive ideals of $A$ have been classified by Joseph $[\mathbf{2 1} ; \mathbf{2 2} ; \mathbf{2 3}$, Chapters 9,10$]$, confirming a conjectured description by Hodges and Levasseur which they had shown to be valid in the case $G=S L_{n}(\mathbb{C})[\mathbf{1 5} ; \mathbf{1 6}]$. Joseph's description for the case of an algebraically closed base field can be summarized as follows. Let $H$ be a maximal torus in $G$ and let $W$ be the associated Weyl group. There is a canonical action of the representation group $H$ on the Hopf algebra $A$ (see (5.2) below), and the orbits in $\operatorname{prim} A$ (the space of primitive ideals of $A$ ) under these automorphisms are parametrized by the elements of $W \times W[\mathbf{2 3}, 10.3 .8]$; we write $\operatorname{prim} A=\bigcup\left\{\operatorname{prim}_{w} A \mid w \in W \times W\right\}$. If $w=\left(w_{+}, w_{-}\right) \in W \times W$ and $P \in \operatorname{prim}_{w} A$, then the factor algebra $A / P$ has Gel'fand-Kirillov dimension $\ell\left(w_{+}\right)+\ell\left(w_{-}\right)+s(w)$, where $s(w)$ denotes the minimum length of an expression for $w_{-}^{-1} w_{+}$as a product of reflections [23, 10.3.9]. Further, it follows from $\left[\mathbf{2 3}, 10.3 .3,10.3 .4\right.$, A.1.18] that $H / \operatorname{Stab}_{H}(P)$ is a torus of rank $(\operatorname{rank} G)-s(w)(\operatorname{cf} .[\mathbf{1 6}, 4.1])$. The classification of $\operatorname{prim} A$ extends to the prime ideals also: one has spec $A=\bigcup\left\{\operatorname{spec}_{w} A \mid w \in W \times W\right\}$. Each $\operatorname{spec}_{w} A$ is nonempty, and if $P \in \operatorname{spec}_{w} A$ then $P$ is an intersection of ideals from $\operatorname{prim}_{w} A$; see $[\mathbf{2 3}, 10.3 .5]$ and (1.3). 
0.4. The classification of $\operatorname{prim} A$ as above is a quantum analog of the following structure on $G$. There is a canonical ("Sklyanin-Drinfel'd") Poisson bracket on $\mathcal{O}(G)$ with respect to which $G$ is a Poisson Lie group [8, Ex. 3.2]. Recall that a symplectic leaf of a Poisson variety is a maximal connected symplectic submanifold [6, $§ 11.1]$. The group $H$ permutes the symplectic leaves of $G$, and the orbits under this action are indexed by the elements of $W \times W$; if $w \in W \times W$ and $\mathcal{A}$ is a leaf of type $w$, then $H / \operatorname{Stab}_{H}(\mathcal{A})$ is a torus of $\operatorname{rank}(\operatorname{rank} G)-s(w)[\mathbf{1 5}$, A.3.2].

0.5. In Sections $1-3$ of this paper we study an arbitrary noetherian algebra $A$ over a field $\mathbb{K}$ satisfying seven hypotheses which are listed in (1.1), (1.2). The most crucial of these are abstracted from the properties of spec $R_{q}[G]$ which are sketched above in (0.3); indeed, as will be shown in Section 5, they are all satisfied when $A=R_{q}[G]$, provided the latter algebra is defined over an algebraically closed field $\mathbb{K}$. (This hypothesis on the field is required for only a part of our results - details are given at appropriate points.) We also show that these hypotheses are satisfied by the quantized function algebras $\mathcal{O}_{\left(q_{i j}\right)}\left(\mathbb{K}^{n}\right)$ when $\mathbb{K}$ is algebraically closed and the multiplicative group $\left\langle q_{i j}\right\rangle$ is torsionfree (Section 4).

Our results in the abstract setting can be summarized as follows.

Theorem. Let $A$ be a noetherian algebra over a field $\mathbb{K}$ which satisfies Assumptions 1-7 of (1.1), (1.2). In particular, there is a disjoint partition $\operatorname{spec} A=$ $\bigcup_{w \in \mathcal{W}} \operatorname{spec}_{w} A$ and there is an abelian subgroup $\mathcal{H}$ of Aut $A$, such that for each $w \in \mathcal{W}$, $\operatorname{spec}_{w} A$ contains a unique minimal member, $J_{w}$, which is $\mathcal{H}$-invariant and $\mathcal{H}$-polynormal $(c f .(0.8))$, and the intersection of the $\mathcal{H}$-orbit of each prime in $\operatorname{spec}_{w} A$ is $J_{w}$.

(a) A satisfies normal separation (cf. (1.6)), and (hence) the strong second layer property.

(b) Fix $w \in \mathcal{W}$. There are subgroups $N_{w} \subseteq T_{w}$ of $\mathcal{H}$, and a finite set $\Sigma_{w} / N_{w}$ of cosets of $T_{w} / N_{w}$ with $T_{w}=\left\langle\Sigma_{w} \cup N_{w}\right\rangle$, with the following properties:

(i) Let $P \in \operatorname{prim}_{w} A$. Then clique $(P)=\left\{\tau(P) \mid \tau \in T_{w}\right\}$ and $N_{w}=\operatorname{Stab}_{\mathcal{H}}(P)$. In particular, clique $(P) \subseteq \operatorname{prim}_{w} A$ and clique $(P)$ is in bijection with $T_{w} / N_{w}$.

(ii) Let $Q \in \operatorname{spec}_{w} A$, and define $T_{Q}=\{\tau \in \mathcal{H} \mid \tau(Q) \in \operatorname{clique}(Q)\}$ and $N_{Q}=$ $\operatorname{Stab}_{\mathcal{H}}(Q)$. Then $N_{w} \subseteq N_{Q}$ and $T_{Q}=T_{w} N_{Q}$ (hence, $T_{Q} / N_{Q}$ is a homomorphic image of $\left.T_{w} / N_{w}\right)$. Moreover, $\operatorname{clique}(Q)=\left\{\tau(Q) \mid \tau \in T_{Q}\right\} \subseteq \operatorname{spec}_{w} A$, so that clique $(Q)$ is in bijection with $T_{Q} / N_{Q}$.

(iii) Nonzero primes $Q, Q^{\prime} \in \operatorname{spec}_{w} A$ satisfy $Q^{\prime} \rightsquigarrow Q$ if and only if $Q^{\prime}=\sigma(Q)$ for some $\sigma \in \Sigma_{w}$.

(iv) Under an additional assumption, the groups $T_{Q} / N_{Q}$ (and in particular $\left.T_{w} / N_{w}\right)$ are free abelian of finite rank, and so the cliques in $\operatorname{spec} A$ are each either singletons or infinite.

These results are proved below in (1.7), (1.9), (2.11), (3.3), and (3.4).

The axiomatic approach we have used was undertaken at the urging of the referee. It facilitated the extension of our original results from the case $G=S L_{n}(\mathbb{C})$ to the general case, and has - we hope - made the development clearer.

0.6. In Section 5 we show that the above theorem applies when the algebra $A=$ $R_{q}[G]$ is defined over an algebraically closed field. Here, $\mathcal{W}=W \times W$ and the abelian group $\mathcal{H}$ consists of winding automorphisms which act semisimply on $A$; further, the subgroups $T_{w} \subseteq \mathcal{H}$ are generated (modulo $N_{w}$ ) by automorphisms 
whose eigenvalues are powers of $q$. The latter property verifies the additional hypothesis of part (iv) of Theorem 0.5 , so that each clique in $R_{q}[G]$ is either a singleton or infinite, and $(\operatorname{rank} G)-s(w)$ is shown to be an upper bound for the rank of the free abelian group $T_{w} / N_{w}$, for all $w \in \mathcal{W}$.

0.7. At the geometric level, the results of this paper lend support to the philosophy $[38,15,16]$ that the structure and representation theory of $R_{q}[G]$ should reflect the Poisson structure of $G$. To explain this statement requires some further background (which is not needed in the rest of the paper). We first recall the construction of the Manin triple corresponding to the Poisson Lie group $G$ [8, p. 802]. Thus, let $\mathbf{g}$ be the Lie algebra of $G$ and

$$
\begin{aligned}
\mathbf{g}_{d} & =\{(x, x) \mid x \in \mathbf{g}\}, & \mathbf{a} & =\{(y,-y) \mid y \in \mathbf{h}\}, \\
\mathbf{d} & =\mathbf{g} \oplus \mathbf{g}, & \mathbf{u}^{+} & =\left\{(x, y) \mid x \in \mathbf{n}^{+}, y \in \mathbf{n}^{-}\right\},
\end{aligned}
$$

where $\mathbf{g}=\mathbf{n}^{-} \oplus \mathbf{h} \oplus \mathbf{n}^{+}$is a triangular decomposition of $\mathbf{g}$. The Iwasawa decomposition of $\mathbf{d}[\mathbf{7}, 1.13 .14]$ corresponding to this data is

$$
\mathbf{d}=\mathbf{g}_{d} \oplus \mathbf{a} \oplus \mathbf{u}^{+} .
$$

The bilinear form on $\mathbf{d}$ given by

$$
\langle(x, y),(z, t)\rangle:=\frac{1}{2}\left((x, z)_{K}-(y, t)_{K}\right),
$$

where $(\cdot, \cdot)_{K}$ is the Killing form on $\mathbf{g}$, is non-degenerate, invariant and symmetric. Set $\mathbf{g}_{r}=\mathbf{a} \oplus \mathbf{u}^{+}$, a solvable algebraic subalgebra of $\mathbf{d}$. One checks easily that $\mathbf{g}_{d}$ and $\mathbf{g}_{r}$ are maximal isotropic subalgebras of $\mathbf{d}$ for $\langle\cdot, \cdot\rangle$; in short,

$$
\left(\mathbf{d}, \mathbf{g}_{d}, \mathbf{g}_{r}\right)
$$

is a Manin triple. Notice that $\mathbf{g}_{d} \cong \mathbf{g}_{r}^{*}$ and $\mathbf{g}_{r} \cong \mathbf{g}_{d}^{*}$; the Lie bracket on $\mathbf{g}_{r}$ is exactly the linearization at 1 of the Poisson structure on $G[\mathbf{2 9}$, p. 504]. (One can also arrive at $\mathbf{g}_{r}$ by using the classical $r$-matrix $R \in \operatorname{End}_{\mathbb{C}}(\mathrm{g})$ corresponding to the Poisson structure on $G$ to define a new Lie bracket $[-,-]_{R}$ on $\mathbf{g}$, setting $[x, y]_{R}=[R x, y]+[x, R y]$; see, for example, $\left.[\mathbf{3 6}].\right)$

Recall next that the space of primitive ideals of the enveloping algebra $U\left(\mathbf{g}_{r}\right)$ is in bijection with the orbits in $\mathbf{g}_{r}^{*}$ under the action of the "adjoint algebraic group" of $\mathbf{g}_{r}[\mathbf{1}]$, and that these orbits are the symplectic leaves for the Poisson structure on $\mathbf{g}_{r}^{*}$ dual to the Lie bracket on $\mathbf{g}_{r}[\mathbf{1 4}, \mathrm{IV} .7 .2 ; \mathbf{2 6}, \S \S 15.1,15.2]$ (cf. $[\mathbf{6}, \S 11.5]$ ). Moreover, $U\left(\mathbf{g}_{r}\right)$ is a quantization (in the sense of $[\mathbf{8}, \S 2]$ ) of the coordinate ring of $\mathbf{g}_{r}^{*}$ equipped with this Poisson structure $[\mathbf{6}, \S 11.6]$.

Compare the above with the situation for $R_{q}[G]$ : This algebra is a quantization of $\mathcal{O}(G)$ where the latter is given the Poisson structure of (0.4). One expects, therefore, that there should be a close resemblance between the algebras $U\left(\mathbf{g}_{r}\right)$ and $R_{q}[G]$. That this is so as regards the cliques and link graphs of the algebras can be seen by comparing the results of this paper with $[\mathbf{3}, \mathbf{2}]$, in which similar results were obtained for enveloping algebras of solvable Lie algebras. The details of this comparison are summarized for the case $G=S L_{2}(\mathbb{C})$ in Example 6.8. 
0.8. All rings, algebras, modules, ring homomorphisms, etc. in this paper are assumed to be unital. We write $J(R)$ for the Jacobson radical of a ring $R$, and $Z(R)$ for its center. As mentioned above, we denote by $\operatorname{spec} R$ the prime spectrum (i.e., the set of prime ideals) of a ring $R$, and by $\operatorname{prim} R$ the set of primitive ideals; similarly, $\max R$ denotes the set of maximal ideals.

A normal element in a ring $R$ is an element $c \in R$ such that $c R=R c$. In case there exists an automorphism $h$ of $R$ such that $c r=h(r) c$ for all $r \in R$, we say that $c$ is $h$-normal. (Such an automorphism need not exist, nor need it be unique, unless $c$ is a regular element.) When we do not wish to record the particular automorphism $h$, but only that it exists in some specified subgroup $H$ of Aut $R$, we say that $c$ is $H$-normal.

A polynormal sequence (or normalizing sequence) in $R$ is a sequence $c_{1}, c_{2}, \ldots, c_{m}$ of elements of $R$ such that each $c_{j}$ is normal modulo the ideal $\left\langle c_{1}, \ldots, c_{j-1}\right\rangle$. In particular, $c_{1}$ must be a normal element of $R$. Such a sequence is called $H$-polynormal (for a subgroup $H$ of Aut $R$ ) if there exist $h_{1}, \ldots, h_{m} \in H$ such that each $c_{j}$ is $h_{j}$-normal modulo $\left\langle c_{1}, \ldots, c_{j-1}\right\rangle$, meaning that $c_{j} r \equiv h_{j}(r) c_{j}\left(\bmod \left\langle c_{1}, \ldots, c_{j-1}\right\rangle\right)$ for all $r \in R$. (A priori, the ideal $\left\langle c_{1}, \ldots, c_{j-1}\right\rangle$ need not be invariant under $h_{j}$, although in practice that will usually be the case.) Finally, an ideal is polynormal (or $H$-polynormal) if it has a polynormal ( $H$-polynormal) sequence of generators.

Any unexplained ring-theoretic terminology or notation is standard, and may be found in $[\mathbf{1 3}]$ or $[\mathbf{3 1}]$.

\section{The Setup: Assumptions and Basic Consequences}

1.1. As basic data, we take

- A noetherian algebra $A$ over a field $\mathbb{K}$;

- An abelian subgroup $\mathcal{H} \subseteq$ Aut $A$;

- A disjoint partition $\operatorname{spec} A=\bigcup^{\bullet}{ }_{w \in \mathcal{W}} \operatorname{spec}_{w} A$;

satisfying the following six assumptions:

Assumption 1. The algebra $A$ is a Jacobson ring, i.e., every prime factor ring of A has zero Jacobson radical.

This assumption is automatically satisfied in case $A$ is countably generated and $\mathbb{K}$ is uncountable (see $[\mathbf{3 1}, 9.1 .8]$ ).

Assumption 2. For each $w \in \mathcal{W}$, there is a (unique) minimum element $J_{w} \in$ $\operatorname{spec}_{w} A$.

Assumption 3. Each of the ideals $J_{w}$ is $\mathcal{H}$-invariant and $\mathcal{H}$-polynormal.

Assumption 4. For each $w \in \mathcal{W}$, there is a multiplicative set $E_{w} \subseteq A / J_{w}$, generated by normal elements, such that the prime ideals of $A / J_{w}$ disjoint from $E_{w}$ are precisely those which come from $\operatorname{spec}_{w} A$, that is,

$$
\left\{P / J_{w} \mid P \in \operatorname{spec}_{w} A\right\}=\left\{Q \in \operatorname{spec} A / J_{w} \mid Q \cap E_{w}=\varnothing\right\} .
$$

Since $E_{w}$ consists of normal elements, it is an Ore set, and so we can form the localization $A_{w}:=\left(A / J_{w}\right)\left[E_{w}^{-1}\right]$. Included in our assumption is that $J_{w} / J_{w}$ must be disjoint from $E_{w}$. Hence, $0 \notin E_{w}$ and so $A_{w} \neq 0$. Since $J_{w}$ is a prime ideal, $A_{w}$ is a prime ring and $A / J_{w}$ embeds in $A_{w}[\mathbf{1 3}, 9.20,9.21]$. Further, standard localization theory shows that extension and contraction give inverse bijections between $\operatorname{spec}_{w} A$ and spec $A_{w}[\mathbf{3 1}, 2.1 .16($ vii); 13, 9.22]. 
Assumption 5. Each $E_{w}$ is generated by a finite set of $\mathcal{H}$-eigenvectors.

It follows from this and Assumption 4 that $\operatorname{spec}_{w} A$ is a union of $\mathcal{H}$-orbits. Further, the multiplicative set $\mathbb{K}^{\times} \cdot E_{w}$ is invariant under $\mathcal{H}$, and so we obtain an induced action of $\mathcal{H}$ on $A_{w}$.

Assumption 6. Each prime ideal of any $A_{w}$ is minimal over a centrally generated semiprime ideal.

It follows, in particular, that spec $A_{w}$ is centrally separated in that for any comparable primes $P<Q$ in $\operatorname{spec} A_{w}$, we have $P \cap Z\left(A_{w}\right)<Q \cap Z\left(A_{w}\right)$. Assumption 6 of course holds in case the primes of each $A_{w}$ are centrally generated, which will be the case for some of the examples we consider.

1.2. While Assumptions 1-6 suffice for some of our initial results, our main theorems require one further hypothesis, which we do not uniformly invoke until Section 3 .

Assumption 7. For each $w \in \mathcal{W}$, the set $\operatorname{prim}_{w} A:=\operatorname{prim} A \cap \operatorname{spec}_{w} A$ equals a single $\mathcal{H}$-orbit.

In particular, $\mathcal{H}$ acts transitively on $\operatorname{prim}_{w} A$. That $\operatorname{prim}_{w} A$ is stable under $\mathcal{H}$ is already guaranteed by Assumptions 4 and 5 , which imply that $\operatorname{spec}_{w} A$ is $\mathcal{H}$-stable.

It is typically more difficult to satisfy Assumption 7 than the other six. For instance, the commutative polynomial ring $A=\mathbb{K}\left[x_{1}, \ldots, x_{n}\right]$, the trivial group $\mathcal{H}=\{\operatorname{id}\} \subseteq$ Aut $A$, and the trivial partition $\operatorname{spec} A=\operatorname{spec}_{w} A$ with a single index $w$ satisfy Assumptions 1-6. A more natural choice of $\mathcal{H}$ for this algebra, which also works for quantum coordinate rings of affine spaces, is presented in Section 4. In that setup, Assumption 7 only holds when $\mathbb{K}$ is algebraically closed.

1.3. Proposition. Let $w \in \mathcal{W}$, and suppose only that Assumptions 1-5 hold.

(a) Each prime in $\operatorname{spec}_{w} A$ is an intersection of primitive ideals from $\operatorname{prim}_{w} A$.

(b) Every maximal element of $\operatorname{spec}_{w} A$ is primitive.

(c) If $P \in \operatorname{prim}_{w} A$, then $P A_{w} \in \operatorname{prim} A_{w}$.

(d) The localization $A_{w}$ is a Jacobson ring.

Proof. Set $R=A / J_{w}$, and choose a finite set of generators for $E_{w}$, say $e_{1}, \ldots, e_{n}$. Since the $e_{j}$ are normal, any prime of $R$ which meets $E_{w}$ must contain some $e_{j}$, and hence must contain the element $e=e_{1} e_{2} \cdots e_{n}$. Consequently,

$$
\left\{P / J_{w} \mid P \in \operatorname{spec}_{w} A\right\}=\{Q \in \operatorname{spec} R \mid e \notin Q\},
$$

because of Assumption 4.

(a) If $P \in \operatorname{spec}_{w} A$, then $P=\bigcap_{i \in I} P_{i}$ for some collection of primitive ideals $P_{i}$, by Assumption 1. Write $I=I^{\prime} \cup I^{\prime \prime}$ where $e \in P_{i} / J_{w}$ for $i \in I^{\prime}$ and $e \notin P_{i} / J_{w}$ for $i \in I^{\prime \prime}$. Then $P=\left(\bigcap_{i \in I^{\prime}} P_{i}\right) \cap\left(\bigcap_{i \in I^{\prime \prime}} P_{i}\right)$, with the first intersection properly containing $P$. Hence, $P=\bigcap_{i \in I^{\prime \prime}} P_{i}$ with $P_{i} \in \operatorname{prim}_{w} A$ for all $i \in I^{\prime \prime}$.

(b) This is immediate from part (a).

(c) Let $M$ be a faithful simple left $(A / P)$-module. Since $J_{w} \subseteq P$, we may also view $M$ as a left $R$-module. For any $x \in E_{w}$ and any nonzero $m \in M$, we have $x \notin P / J_{w}=\operatorname{ann}_{R} M$, whence $R x m=x R m=x M \neq 0$ and so $x m \neq 0$. Hence, $M$ is $E_{w}$-torsionfree. It follows that the localization $\left[E_{w}^{-1}\right] M$ is a simple left $A_{w^{-}}$ module $[13,9.18]$ and that $M$ embeds in $\left[E_{w}^{-1}\right] M$. Hence, $Q=\operatorname{ann}_{A_{w}}\left[E_{w}^{-1}\right] M$ is 
a primitive ideal of $A_{w}$ and $Q \cap R \subseteq P / J_{w}$. On the other hand, $P\left[E_{w}^{-1}\right] M=0$ because $P A_{w}=A_{w} P$ (cf. $[\mathbf{1 3}, 9.20]$ ). Thus $Q \cap R=P / J_{w}$, and so $P A_{w}=Q[\mathbf{1 3}$, 9.22]. Therefore $P A_{w} \in \operatorname{prim} A_{w}$.

(d) Consider an arbitrary prime $P A_{w} \in \operatorname{spec} A_{w}$, where $P \in \operatorname{spec}_{w} A$. By parts (a) and (c), $P=\bigcap_{i \in I} P_{i}$ for some primitive ideals $P_{i} \in \operatorname{prim}_{w} A$, and each $P_{i} A_{w} \in \operatorname{prim} A_{w}$. For $i \in I$, the prime $P_{i} / J_{w}$ in $R$ is disjoint from $E_{w}$, and so $A / P_{i}$ is $E_{w}$-torsionfree $[\mathbf{1 3}, 9.21]$. It follows that $\bigcap_{i \in I} P_{i} A_{w}=P A_{w}$, and therefore $J\left(A_{w} / P A_{w}\right)=0$.

1.4. A noetherian $\mathbb{K}$-algebra $R$ is said to satisfy the Nullstellensatz (over $\mathbb{K}$ ) provided that $R$ is a Jacobson ring and the endomorphism ring of every simple $R$ module is algebraic over $\mathbb{K}[\mathbf{3 1}, 9.1 .4]$. These conditions are, in particular, satisfied when $R$ is countably generated and $\mathbb{K}$ is uncountable [31, 9.1.8].

Corollary. Suppose that A satisfies the Nullstellensatz.

(a) For each $w \in \mathcal{W}$, the bijection of $\operatorname{spec}_{w} A$ onto spec $A_{w}$ given by $P \mapsto P A_{w}$ restricts to a bijection of $\operatorname{prim}_{w} A$ onto max $A_{w}$. In other words, $\operatorname{prim}_{w} A$ equals the set of maximal elements of $\operatorname{spec}_{w} A$.

(b) Assumption 7 holds if and only if $\mathcal{H}$ acts transitively on $\max A_{w}$ for each $w \in \mathcal{W}$.

Proof. (a) If $P \in \operatorname{spec}_{w} A$ and $P A_{w} \in \max A_{w}$, then $P$ must be maximal in $\operatorname{spec}_{w} A$, and so $P \in \operatorname{prim}_{w} A$ by Proposition $1.3(\mathrm{~b})$.

Conversely, let $P \in \operatorname{prim}_{w} A$, and let $M$ be a faithful simple left $(A / P)$-module. We adopt the notation of the proof of Proposition 1.3(c). As shown in that proof, the localization $\left[E_{w}^{-1}\right] M$ is a simple left $A_{w}$-module, $M$ embeds in $\left[E_{w}^{-1}\right] M$, and the ideal $Q=\operatorname{ann}_{A_{w}}\left[E_{w}^{-1}\right] M$ coincides with $P A_{w}$. Now the image of $M$ in $\left[E_{w}^{-1}\right] M$, being simple and essential, must be the unique simple $R$-submodule of $\left[E_{w}^{-1}\right] M$. Hence, restriction from $\left[E_{w}^{-1}\right] M$ to this image yields a $\mathbb{K}$-algebra embedding $\operatorname{End}_{A_{w}}\left[E_{w}^{-1}\right] M \hookrightarrow \operatorname{End}_{A} M$. Consequently, $Z\left(A_{w}\right) /\left(Q \cap Z\left(A_{w}\right)\right)$ embeds in $\operatorname{End}_{A} M$. It now follows from the Nullstellensatz that $Z\left(A_{w}\right) /\left(Q \cap Z\left(A_{w}\right)\right)$ is algebraic over $\mathbb{K}$. This commutative domain must therefore be a field, and so $Q \cap Z\left(A_{w}\right)$ must be a maximal ideal of $Z\left(A_{w}\right)$. We thus conclude from Assumption 6 that $P A_{w}=Q$ is a maximal ideal of $A_{w}$.

(b) This is clear from part (a).

1.5. Corollary. Assumption 7 implies statement 1.4(a).

Proof. The maximal elements of $\operatorname{spec}_{w} A$ lie in $\operatorname{prim}_{w} A$ by Proposition 1.3(b). In particular, at least one element of $\operatorname{prim}_{w} A$ is maximal in $\operatorname{spec}_{w} A$. Since $\mathcal{H}$ acts transitively on $\operatorname{prim}_{w} A$ (Assumption 7), all elements of $\operatorname{prim}_{w} A$ must be maximal in $\operatorname{spec}_{w} A$.

The natural companion result to Corollary $1.5-$ that $\operatorname{prim} A_{w}=\max A_{w}-\operatorname{does}$ not seem to follow from our assumptions. It can be shown to hold in the examples we consider later, but since we shall not need this result, we will leave the details to the reader.

1.6. We say that the prime spectrum of a ring $R$ has normal separation provided that for any pair of comparable primes $P<Q$ in spec $R$, the factor $Q / P$ contains a nonzero normal element of $R / P$. Normal separation is connected to the second layer condition via the Artin-Rees property, as follows. 
An ideal $I$ in a ring $R$ satisfies the left Artin-Rees property if for each left ideal $L$ of $R$, there is a positive integer $n$ such that $L \cap I^{n} \subseteq I L$. This is equivalent to the condition that every finitely generated left $R$-module which has an essential submodule annihilated by $I$ must be annihilated by a power of $I[\mathbf{1 3}, 11.11 ; \mathbf{3 1}$, 4.2.2]. The right AR-property is defined symmetrically, and an AR-ideal is an ideal satisfying the left and right AR-properties. Any normal element in a noetherian ring $R$ generates an AR-ideal [31, 4.1.10]. Hence, if spec $R$ has normal separation, then it is also $A R$-separated in the sense that for any comparable primes $P<Q$ in $R$, the ideal $Q / P$ in $R / P$ contains a nonzero AR-ideal. Now AR-separation implies the strong second layer condition $[\mathbf{2 0}, 8.1 .7 ; \mathbf{1 3}, 11.14]$, and therefore normal separation implies this condition.

1.7. Proposition. spec $A$ has normal separation, and therefore $A$ satisfies the strong second layer condition.

Proof. As noted in (1.6), the strong second layer condition follows from normal separation.

Consider primes $P<Q$ in $\operatorname{spec} A$. Then $P \in \operatorname{spec}_{w} A$ for some $w \in \mathcal{W}$, and $J_{w} \subseteq P$. If $Q \notin \operatorname{spec}_{w} A$, there exists an element $c \in\left(Q / J_{w}\right) \cap E_{w}$, and the image of $c$ in $A / P$ is a nonzero normal element lying in $Q / P$. If $Q \in \operatorname{spec}_{w} A$, then by Assumption 6 there exists an element $z \in Z\left(A_{w}\right) \cap Q A_{w} \backslash P A_{w}$. Further, $z=c d^{-1}$ for some $c \in Q / J_{w}$ and $d \in E_{w}$. Since $d$ is normal in $A / J_{w}$, it follows from the centrality of $z$ that $c$ is normal in $A / J_{w}$. Thus the image of $c$ in $A / P$ is a nonzero normal element contained in $Q / P$.

Therefore spec $A$ has normal separation.

1.8. Given a set $\Phi$ of automorphisms of a ring $R$ and an ideal $P$ in $R$, let $(P: \Phi)$ denote the largest $\Phi$-ideal $(\Phi$-invariant ideal) contained in $P$. In case $\Phi$ is a subgroup of Aut $R$, the ideal $(P: \Phi)$ equals the intersection of the ideals in the $\Phi$-orbit of $P$.

It is well known that if $P$ is prime, then $(P: \Phi)$ is $\Phi$-prime. Namely, if $I_{1}$ and $I_{2}$ are $\Phi$-ideals whose product is contained in $(P: \Phi)$, then $I_{1} I_{2} \subseteq P$ and so some $I_{j} \subseteq P$; since $I_{j}$ is $\Phi$-invariant, it must be contained in $(P: \Phi)$.

Now assume that $R$ is noetherian and $\Phi$ is a subgroup of Aut $R$. In this case, a standard argument shows that the primes minimal over any $\Phi$-prime ideal $J$ form a single $\Phi$-orbit, and that $J$ equals the intersection of this orbit, i.e., $J=(P: \Phi)$ for any prime $P$ minimal over $J$. In particular, $J$ must be semiprime. (Cf. $[\mathbf{9}$, Remarks $4^{*}, 5^{*}$, p. $\left.338 ; 35,14.2\right]$.) To see this, choose one prime $P_{i}$ from each of the distinct $\Phi$-orbits of primes minimal over $J$. There are finitely many $P_{i}$, and the intersection of the ideals $\left(P_{i}: \Phi\right)$ equals the prime radical of $J$. Since $J$ contains some power of the product of the $\left(P_{i}: \Phi\right)$, it must contain some $\left(P_{j}: \Phi\right)$. Therefore $J=\left(P_{j}: \Phi\right)$.

1.9. Proposition. Suppose that Assumption 7 holds.

(a) For $P \in \operatorname{spec} A$ and $w \in \mathcal{W}$, we have $P \in \operatorname{spec}_{w} A$ if and only if the intersection of the $\mathcal{H}$-orbit of $P$ equals $J_{w}$.

(b) The map $w \mapsto J_{w}$ gives a bijection from $\mathcal{W}$ onto the set of prime $\mathcal{H}$-ideals of $A$.

(c) The prime $\mathcal{H}$-ideals of $A$ coincide with the $\mathcal{H}$-prime ideals.

Proof. (a) By Assumption 7, $\operatorname{prim}_{w} A$ consists of a single $\mathcal{H}$-orbit, and so $(Q: \mathcal{H})=$ $\cap \operatorname{prim}_{w} A$ for any $Q \in \operatorname{prim}_{w} A$. Since every prime in $\operatorname{spec}_{w} A$ is an intersection of 
primes from $\operatorname{prim}_{w} A$ (Corollary 1.5), and since $J_{w}$ is $\mathcal{H}$-invariant, it follows that $(Q: \mathcal{H})=J_{w}$ for all $Q \in \operatorname{spec}_{w} A$.

Conversely, assume that $(P: \mathcal{H})=J_{w}$. Since the elements of $E_{w}$ are nonzero $\mathcal{H}$-eigenvectors in $A / J_{w}$, we must have $\left(P / J_{w}\right) \cap E_{w}=\varnothing$. Therefore $P \in \operatorname{spec}_{w} A$ by Assumption 5 .

(b) The ideals $J_{w}$ are clearly prime $\mathcal{H}$-ideals. On the other hand, any prime $\mathcal{H}$-ideal $J$ equals the intersection of its own $\mathcal{H}$-orbit. Moreover, $J \in \operatorname{spec}_{w} A$ for some $w$, and thus $J=J_{w}$ by part (a). Finally, $J_{w} \neq J_{y}$ for distinct $w, y \in \mathcal{W}$ because $\operatorname{spec}_{w} A$ and $\operatorname{spec}_{y} A$ are disjoint.

(c) Obviously prime $\mathcal{H}$-ideals are $\mathcal{H}$-prime. If $J$ is an $\mathcal{H}$-prime ideal of $A$, then by $(1.8), J=(P: \mathcal{H})$ for some prime $P$. But $P \in \operatorname{spec}_{w} A$ for some $w \in \mathcal{W}$, and $(P: \mathcal{H})=J_{w}$ by part (a). Therefore $J=J_{w}$ is prime.

Proposition 1.9 allows us to rewrite the $\operatorname{spec}_{w}$-partition of $\operatorname{spec} A$ solely in terms of the action of $\mathcal{H}$, in the following fashion:

1.10. Theorem. Suppose that Assumption 7 holds. For each prime $\mathcal{H}$-ideal $J$ in $A$, let $E_{J}$ denote the set of nonzero normal $\mathcal{H}$-eigenvectors in $A / J$, and set

$$
\operatorname{spec}_{J} A=\left\{P \in \operatorname{spec} A \mid J \subseteq P \text { and }(P / J) \cap E_{J}=\varnothing\right\} .
$$

(a) $\operatorname{spec} A$ is the disjoint union of the sets $\operatorname{spec}_{J} A$.

(b) The set of primitive ideals in each $\operatorname{spec}_{J} A$ coincides with the set of maximal elements of $\operatorname{spec}_{J} A$, and $\mathcal{H}$ acts transitively on this set.

(c) A prime $P \in \operatorname{spec} A$ lies in $\operatorname{spec}_{J} A$ if and only if $(P: \mathcal{H})=J$.

Proof. (c) By Proposition 1.9, $J=J_{w}$ for some $w \in \mathcal{W}$, and we note that $E_{w} \subseteq E_{J}$. If $P \in \operatorname{spec}_{J} A$, then $P \in \operatorname{spec}_{w} A$ by Assumption 4, and so $(P: \mathcal{H})=J$ by Proposition 1.9. Conversely, if $(P: \mathcal{H})=J$, then $J \subseteq P$ and $P / J$ contains no nonzero $\mathcal{H}$-eigenvectors, whence $(P / J) \cap E_{J}=\varnothing$ and so $P \in \operatorname{spec}_{J} A$.

Statements (a) and (b) now follow from part (c) together with Proposition 1.9, Corollary 1.5, and Assumption 7.

\section{Linked PRime Ideals}

We continue with the setup and assumptions of (1.1); Assumption 7 is not needed in this section. This section is devoted to the relationship between links and automorphisms in $A$. We show first that the cliques in $\operatorname{spec} A$ are contained within the sets $\operatorname{spec}_{w} A$, that is, each $\operatorname{spec}_{w} A$ is closed under incoming and outgoing links. Next, we show that all non-minimal primes in spec $A$ have self-links. The remainder of the section is devoted to proving that all links in spec $A$ arise from particular automorphisms of $A$; further, links are "generic" in the sense that all links in $\operatorname{spec}_{w} A$ lift to links in $\operatorname{prim}_{w} A$ given by the same automorphisms.

2.1. Recall that if $P$ and $Q$ are linked primes in a noetherian ring, then any ARideal contained in one of them must be contained in the other $[\mathbf{2 0}, 5.3 .10 ; \mathbf{1 3}$, 11.16]. Thus, the presence of AR-ideals imposes a certain rigidity on the graph of links. 
2.2. Lemma. For any $w \in \mathcal{W}$, the ideal $J_{w}$ is an $A R$-ideal. Further, if $c+J_{w} \in E_{w}$, then $J_{w}+A c$ is an AR-ideal.

Proof. By Assumption $3, J_{w}$ has a sequence of generators $x_{1}, \ldots, x_{m}$ such that each $x_{j}$ is $\mathcal{H}$-normal modulo $\left\langle x_{1}, \ldots, x_{j-1}\right\rangle$. Since $J_{w}$ is $\mathcal{H}$-invariant, it follows that

$$
x_{j} J_{w}+\left\langle x_{1}, \ldots, x_{j-1}\right\rangle=J_{w} x_{j}+\left\langle x_{1}, \ldots, x_{j-1}\right\rangle
$$

for $j=1, \ldots, m$. Therefore by McConnell's criterion [31, 4.2.7], $J_{w}$ is an AR-ideal.

By Assumptions 4 and $5, c+J_{w}$ is a normal $\mathcal{H}$-eigenvector. Hence, $x_{1}, \ldots, x_{m}, c$ is a polynormal sequence of generators for $J_{w}+A c$, and $J_{w}+A c$ is $\mathcal{H}$-invariant. Thus

$$
x_{j}\left(J_{w}+A c\right)+\left\langle x_{1}, \ldots, x_{j-1}\right\rangle=\left(J_{w}+A c\right) x_{j}+\left\langle x_{1}, \ldots, x_{j-1}\right\rangle
$$

for $j=1, \ldots, m$. Clearly $c\left(J_{w}+A c\right)+J_{w}=\left(J_{w}+A c\right) c+J_{w}$ as well, and therefore $J_{w}+A c$ is an AR-ideal.

2.3. Proposition. If $w \in \mathcal{W}$ and $P \in \operatorname{spec}_{w} A$, then clique $(P) \subseteq \operatorname{spec}_{w} A$.

Proof. It suffices to show that any $Q \in \operatorname{spec} A$ which is linked to or from $P$ must lie in $\operatorname{spec}_{w} A$.

Since $J_{w} \subseteq P$ and $J_{w}$ is AR by Lemma 2.2, we have $J_{w} \subseteq Q$. Suppose there exists a coset $c+J_{w} \in\left(Q / J_{w}\right) \cap E_{w}$; then $J_{w}+A c \subseteq Q$. Since $J_{w}+A c$ is AR (Lemma 2.2), it follows that $J_{w}+A c \subseteq P$, contradicting the fact that $P / J_{w}$ is disjoint from $E_{w}$. Therefore $\left(Q / J_{w}\right) \cap E_{w}=\varnothing$, and hence $Q \in \operatorname{spec}_{w} A$.

2.4. Lemma. For $w \in \mathcal{W}$, there are no links in spec $A_{w}$ except possibly self-links.

Proof. Consider a link $P \rightsquigarrow Q$ in $\operatorname{spec} A_{w}$. By Assumption 6,P is minimal over a centrally generated semiprime ideal $N$, and since $N$ is an AR-ideal it must be contained in $Q$. It follows from $[\mathbf{2 0}, 5.3 .12$ and proof $]$ that either $P=Q$ or $P / N \rightsquigarrow Q / N$ in $A_{w} / N$ (this also follows from Lemma 2.7 below). However, there are no links to or from minimal primes in a semiprime noetherian ring $[\mathbf{1 3}, 11.17]$, and therefore $P=Q$.

2.5. Proposition. Let $R$ be a noetherian ring satisfying the right second layer condition, and $P$ any non-minimal prime of $R$. Then there exists $Q \in \operatorname{spec} R$ such that $Q \rightsquigarrow P$.

Proof. There exists a minimal prime $P_{0}<P$, and any link of the form $Q / P_{0} \rightsquigarrow P / P_{0}$ lifts to a link $Q \rightsquigarrow P$. Hence, there is no loss of generality in assuming that $R$ is a prime ring and $P \neq 0$. Let $E$ be the injective hull of $(R / P)_{R}$. Then $E$ is a faithful right $R$-module $[\mathbf{1 3}, 12.9]$, and so $E_{1}:=\operatorname{ann}_{E}(P)$ is a proper submodule of $E$. Note that $E_{1}$ is a torsionfree $(R / P)$-module. Choose an affiliated submodule $E_{2} / E_{1}$ of $E / E_{1}$, with affiliated prime $Q$. Since $R$ satisfies the right second layer condition, we conclude from Jategaonkar's Main Lemma $[\mathbf{2 0}, 6.1 .3 ; \mathbf{1 3}, 11.1]$ that $Q \rightsquigarrow P$.

2.6. Theorem. Every non-minimal prime in $\operatorname{spec} A$ is linked to itself.

Proof. Let $P$ be a non-minimal prime in $\operatorname{spec} A$. Then $P \in \operatorname{spec}_{w} A$ for some $w \in \mathcal{W}$. If $P A_{w} \neq 0$, then since $A_{w}$ is a prime ring, Proposition 2.5 shows that there must be a prime in spec $A_{w}$ linked to $P A_{w}$. In view of Lemma 2.4, the only possibility is $P A_{w} \rightsquigarrow P A_{w}$, from which it follows that $P \rightsquigarrow P$. 
If $P A_{w}=0$, then $P=J_{w}$. Proposition 2.5 implies the existence of a prime $Q \in \operatorname{spec} A$ linked to $P$, and $Q \in \operatorname{spec}_{w} A$ by Proposition 2.3. But then $P \subseteq Q$, whence $P=Q$ (because of the second layer condition, distinct primes in the same clique are incomparable $[\mathbf{1 3}, 12.6])$. Thus $P \rightsquigarrow P$ in this case also.

Since our assumptions include no restrictions on the prime radical of $A$, the question whether the minimal primes of $A$ are linked to themselves is open. One can easily concoct examples satisfying (1.1) in which the minimal primes do have self-links (e.g., $\mathbb{K}[x] /\left\langle x^{2}\right\rangle$ ). However, the quantum coordinate rings to which we will apply our results are domains. In those cases, 0 is the unique minimal prime of $A$, and 0 cannot be linked to any prime.

The key to writing cliques as orbits lies in the following easy lemma. It is relatively well known, but we have not located a reference for this form of it. The proof below is adapted from $[\mathbf{2}, 4.1]$ and $[\mathbf{2 7}, 2.5]$.

2.7. Lemma. Let $P \rightsquigarrow Q$ be linked prime ideals in a noetherian ring $R$, and $I$ an ideal contained in $P \cap Q$. Suppose that $I$ has a polynormal sequence of generators, say $c_{1}, \ldots, c_{m}$, and that each $c_{j}$ is $\alpha_{j}$-normal modulo $\left\langle c_{1}, \ldots, c_{j-1}\right\rangle$ for some $\alpha_{j} \in$ Aut $R$. Then either $P / I \rightsquigarrow Q / I$ or $P=\alpha_{j}(Q)$ for some $j$.

Proof. Suppose that the link $P \rightsquigarrow Q$ is implemented by a bimodule $(P \cap Q) / B$. If $I \subseteq B$, then $P / I \rightsquigarrow Q / I$ via the bimodule $[(P / I) \cap(Q / I)] /[B / I]$. If $I \nsubseteq B$, let $j$ be the least index such that $c_{j} \notin B$. Then the coset $c=c_{j}+B$ is a nonzero $\alpha_{j}$-normal element of the bimodule $(P \cap Q) / B$. Since this bimodule is torsionfree as a left $(R / P)$-module and as a right $(R / Q)$-module, we conclude that

$$
P=1 \cdot \operatorname{ann}_{R}(R c)=1 \cdot \operatorname{ann}_{R}(c)=\alpha_{j}\left(\mathrm{r} \cdot \operatorname{ann}_{R}(c)\right)=\alpha_{j}\left(\mathrm{r} \cdot \operatorname{ann}_{R}(c R)\right)=\alpha_{j}(Q) .
$$

The results of this section so far can be used to show that links in spec $A$ all arise from the actions of certain automorphisms from $\mathcal{H}$, namely those associated with $\mathcal{H}$-polynormal sequences of generators for the $J_{w}$. This follows from an application of Lemma 2.7 with $R=A$ and $I=J_{w}$. However, we will also need that links in $\operatorname{spec}_{w} A$ lift to corresponding links in $\operatorname{prim}_{w} A$, in the following sense: whenever $P \rightsquigarrow Q$ are distinct linked primes in $\operatorname{spec}_{w} A$, there exists a suitable $h \in \mathcal{H}$ such that $P=h(Q)$ and $h(K) \rightsquigarrow K$ for $K \in \operatorname{prim}_{w} A$. To prove this, we require a stronger version of Lemma 2.7, namely Corollary 2.10, which is a variant of $[\mathbf{2}, 4.1]$.

2.8. It will be convenient to work with bonds between prime factor rings. Recall that a bond $\left[\mathbf{2 0}\right.$, p. 123] from a prime noetherian ring $R^{\prime}$ to a prime noetherian ring $R^{\prime \prime}$ is a nonzero noetherian $R^{\prime}$ - $R^{\prime \prime}$-bimodule which is torsionfree as a left $R^{\prime}$-module and as a right $R^{\prime \prime}$-module. We shall mainly be concerned with bonds between prime factors $R / P$ and $R / Q$ of a given noetherian ring $R$, and in fact with bonds of the form $I / J$ where $I>J$ are ideals of $R$. (Such bonds are sometimes called internal bonds or ideal links; links as defined in (0.1) are a special type of ideal link.)

A well-known argument of Jatagaonkar [20, proof of 8.2.4] shows that if $P$ and $Q$ are primes in a noetherian ring $R$ with the second layer condition, and if there is a bimodule subfactor of $R$ giving a bond from $R / P$ to $R / Q$, then either $P=Q$ or there is a chain of links $P_{0}=P \rightsquigarrow P_{1} \rightsquigarrow \cdots \rightsquigarrow P_{r}=Q$ (cf. [10, 1.1]). The determining factor distinguishing the two cases is how "deep" within the ring $R$ the bond lies, relative to $P \cap Q$. This can be proved by modifying Jategaonkar's argument as follows. 
Theorem. Let $R$ be a noetherian ring satisfying the second layer condition, and let $P, Q \in \operatorname{spec} R$. Suppose that there exist ideals $C \supset D$ in $R$ such that $C / D$ is a bond from $R / P$ to $R / Q$.

(a) If $P \cap Q \cap C \subseteq D$, then $P=Q$.

(b) If $P \cap Q \cap C \nsubseteq D$, then there exists a chain of links

$$
P_{0}=P \rightsquigarrow P_{1} \rightsquigarrow \cdots \rightsquigarrow P_{r}=Q
$$

in $\operatorname{spec} R$ with $r \geq 1$.

Proof. Recall that, because of the bond from $R / P$ to $R / Q$, the rings $R / P$ and $R / Q$ must have the same classical Krull dimension $[\mathbf{1 3}, 12.6]$.

(a) In this case we have $C \cap[(P \cap Q)+D]=D$, and so

$$
[(P \cap Q)+C] /[(P \cap Q)+D] \cong C / D .
$$

Hence, there is no loss of generality in assuming that $P \cap Q \subseteq D$. After passing to $R /(P \cap Q)$, we may assume that $P \cap Q=0$. Since cl.K.dim $R / P=\operatorname{cl} . K . \operatorname{dim} R / Q$, neither $P$ nor $Q$ is properly contained in the other, and so $P$ and $Q$ are both minimal primes. As a result, the bond survives localization with respect to $\mathcal{C}_{R}(0)$, and hence we may assume that $R$ is semisimple. Now the ideal $C$ must be generated by a central idempotent, and we conclude that $P=Q$.

(b) In this case, the ideal factor $(P \cap Q \cap C) /(P \cap Q \cap D)$ is nonzero. Since this factor is isomorphic to a sub-bimodule of $C / D$, it also is a bond from $R / P$ to $R / Q$. Hence, there is no loss of generality in assuming that $C \subseteq P \cap Q$. Then, we may pass to $R / D$, and so we may also assume that $D=0$. Choose an ideal $J$ in $R$ maximal with respect to the property $J \cap C=0$. Then $J \subseteq l \cdot \operatorname{ann}_{R}(C)=P$ and similarly $J \subseteq Q$. Thus we may pass to $R / J$, and hence there is no loss of generality in assuming that $C$ is essential as an ideal of $R$.

Now choose a right ideal $A$ of $R$ maximal with respect to the property $A \cap C=0$. Then $C$ is isomorphic to an essential submodule of $R / A$, whence $Q$ is the unique associated prime of $R / A$, and $A_{1} / A:=\operatorname{ann}_{R / A}(Q)$ is a torsionfree right $(R / Q)$ module. Note that $A_{1} / A$ is essential in $R / A$. Let

$$
0 \subset A_{1} / A \subset A_{2} / A \subset \cdots \subset A_{t} / A=R / A
$$

be an affiliated series for $R / A$, with corresponding affiliated primes $Q_{1}=Q, Q_{2}, \ldots$, $Q_{t}$. Since $A_{1} / A$ is essential in $R / A$, it follows from the (right) second layer condition that each $Q_{j}$ has a chain of links to $Q[\mathbf{2 0}, 9.1 .2 ; \mathbf{1 3}, 11.6]$; further, for $j \geq 2$ there is a chain $Q_{j} \rightsquigarrow \cdots \rightsquigarrow Q$ of positive length. The product $Q_{t} Q_{t-1} \cdots Q_{1}$ annihilates $R / A$, whence $Q_{t} Q_{t-1} \cdots Q_{1} \cap C \subseteq A \cap C=0$. Since $C$ is essential as an ideal of $R$, we obtain $Q_{t} Q_{t-1} \cdots Q_{1}=0$.

Note that $Q_{t} Q_{t-1} \cdots Q_{2} C \subseteq Q_{t} Q_{t-1} \cdots Q_{1}=0$, whence $Q_{t} Q_{t-1} \cdots Q_{2} \subseteq P$. Thus $Q_{j} \subseteq P$ for some $j \geq 2$. There is a chain of links

$$
P_{0}=Q_{j} \rightsquigarrow P_{1} \rightsquigarrow \cdots \rightsquigarrow P_{r}=Q
$$

with $r \geq 1$. Because of these links (and the bond from $R / P$ to $R / Q$ ) we have

$$
\text { cl.K.dim } R / Q_{j}=\text { cl.K.dim } R / Q=\text { cl.K.dim } R / P
$$

$[\mathbf{1 3}, 12.6]$. Therefore $Q_{j}=P$. 
2.9. Lemma. Let $R$ be a noetherian ring satisfying the second layer condition and $I$ an ideal of $R$. Let $K_{0}$ and $K$ be prime ideals of $R$, containing $I$, such that the cliques of $K_{0} / I$ and $K / I$ in $\operatorname{spec} R / I$ are singletons. If there is a bimodule subfactor of $I / I^{2}$ which is a bond from $R / K_{0}$ to $R / K$, then $K_{0} \rightsquigarrow K$.

Proof. Let $I \supseteq C \supset D \supseteq I^{2}$ be ideals such that $C / D$ is a bond from $R / K_{0}$ to $R / K$. After passing to $R / D$, we may assume that $D=0$.

Choose a right ideal $A \subseteq I$ maximal with respect to the property $A \cap C=0$. Then $C$ is isomorphic to an essential submodule of $I / A$. Hence, $K$ is the unique associated prime of $I / A$, and $\operatorname{ann}_{I / A}(K)$ is a torsionfree right $(R / K)$-module. We may view $I / A$ as a right $(R / I)$-module, because $I^{2}=0$. Hence, $I / A$ is annihilated by some finite product of primes from the (right) clique of $K / I$. By hypothesis, the clique of $K / I$ is a singleton, and so $(I / A) K^{m}=0$ for some $m$. Thus $I K^{m} \cap C \subseteq A \cap C=0$.

Now $\left(C+I K^{m}\right) / I K^{m} \cong C$, and so the ideal $\left(C+I K^{m}\right) / I K^{m}$ in the ring $R / I K^{m}$ gives a bond from $R / K_{0}$ to $R / K$. After passing to $R / I K^{m}$, we may assume that $I K^{m}=0$. Since $K_{0} \cap K \supseteq I \supseteq C$, Theorem 2.8 provides us with a chain of links $K_{0} \rightsquigarrow K_{1} \rightsquigarrow \cdots \rightsquigarrow K_{r}=K$ in spec $R$, with $r \geq 1$. Note that cl.K.dim $R / K_{i}=$ cl.K.dim $R / K$ for all $i[\mathbf{1 3}, 12.6]$. If $K_{r-1}=K_{0}$ we are done, so assume that $K_{r-1} \neq K_{0}$.

Let $j$ be the least index such that $K_{j} \neq K_{0}$; then $K_{0} \rightsquigarrow K_{j}$. This link can be given by $\left(K_{0} \cap K_{j}\right) / B$ for some ideal $B \supseteq K_{0} K_{j}$. Since $K_{0} / I \leftrightarrow K_{j} / I$ (by hypothesis), $B$ cannot contain $I$. Hence, $(I+B) / B$ is a nonzero torsionfree right $\left(R / K_{j}\right)$-module. But $K^{m}$ annihilates $(I+B) / B$ on the right, whence $K^{m} \subseteq K_{j}$ and so $K \subseteq K_{j}$. Since $R / K$ and $R / K_{j}$ have the same classical Krull dimension, we conclude that $K=K_{j}$, and therefore $K_{0} \rightsquigarrow K$.

2.10. Corollary. Let $R$ be a noetherian ring satisfying the second layer condition and $I$ an ideal of $R$. Suppose that $I$ has a polynormal sequence of generators, say $c_{1}, \ldots, c_{m}$, and that each $c_{j}$ is $\alpha_{j}$-normal modulo $\left\langle c_{1}, \ldots, c_{j-1}\right\rangle$ for some $\alpha_{j} \in$ Aut $R$. Let $\mathcal{K}$ be a link-closed subset of $\operatorname{spec} R$ which is invariant under $\alpha_{1}, \ldots, \alpha_{m}$. Suppose that each $K \in \mathcal{K}$ contains $I$ and that the clique of $K / I$ in $\operatorname{spec} R / I$ is a singleton for all $K \in \mathcal{K}$.

If $P \rightsquigarrow Q$ are distinct linked primes in $\mathcal{K}$, then there exists an index $j$ such that $P=\alpha_{j}(Q)$ and such that $\alpha_{j}(K) \rightsquigarrow K$ for all $K \in \mathcal{K}$ that contain $Q$.

Proof. Suppose that the link $P \rightsquigarrow Q$ is implemented by $(P \cap Q) / I_{0}$, and note that $I^{2} \subseteq I_{0}$. Since $P / I \leftrightarrow Q / I$ (because $P, Q \in \mathcal{K}$ ), we have $I \nsubseteq I_{0}$. Let $j$ be the least index such that $c_{j} \notin I_{0}$, and set $c=c_{j}+I_{0}$. Then $c$ is a nonzero $\alpha_{j}$-normal element of $\left(I+I_{0}\right) / I_{0}$, and $P=\alpha_{j}(Q)$ as in the proof of Lemma 2.7. Moreover, l.ann $R(c)=P$ and $\mathrm{r} \cdot \mathrm{ann}_{R}(c)=Q$.

Now let $K$ be any prime in $\mathcal{K}$ that contains $Q$. Then $\alpha_{j}(K) \in \mathcal{K}$ as well, and so the cliques of $\alpha_{j}(K) / I$ and $K / I$ in spec $R / I$ are singletons. Note that $I_{0} \subseteq Q \subseteq K$ and $I_{0} \subseteq P=\alpha_{j}(Q) \subseteq \alpha_{j}(K)$, so that $K /\left(I+I_{0}\right)$ and $\alpha_{j}(K) /\left(I+I_{0}\right)$ are primes in $R /\left(I+I_{0}\right)$. The cliques of these primes in spec $R /\left(I+I_{0}\right)$ are singletons. Since r. $\operatorname{ann}_{R}(c)=Q \subseteq K$, we have $c R / c K \cong R / K$ as right $R$-modules. Similarly, l.ann $R(c)=\alpha_{j}(Q) \subseteq \alpha_{j}(K)$, and so $c R / c K=R c / \alpha_{j}(K) c \cong R / \alpha_{j}(K)$ as left $R$-modules. Hence, $c R / c K$ is a bond from $R / \alpha_{j}(K)$ to $R / K$. Since $c R / c K$ is a bimodule subfactor of $\left(I+I_{0}\right) / I_{0}$, we conclude from Lemma 2.9 that $\alpha_{j}(K) \rightsquigarrow K . \square$ 
2.11. We can now prove our fundamental result on the relationship between links and automorphisms in $A$. For each $w \in \mathcal{W}$, choose an $\mathcal{H}$-polynormal sequence of generators $x_{w, 1}, \ldots, x_{w, m(w)}$ for $J_{w}$ (Assumption 3). Thus, each $x_{w, j}$ is $h_{w, j \text {-normal }}$ modulo $\left\langle x_{w, 1}, \ldots, x_{w, j-1}\right\rangle$ for some $h_{w, j} \in \mathcal{H}$. All links in spec $A$ arise from the automorphisms $h_{w, j}$ as follows.

Theorem. Let $P$ and $Q$ be distinct primes in some $\operatorname{spec}_{w} A$. If $P \rightsquigarrow Q$, then $P=h_{w, j}(Q)$ for some $j \in\{1, \ldots, m(w)\}$. Furthermore, $j$ can be chosen so that $h_{w, j}(K) \rightsquigarrow K$ for all $K \in \operatorname{spec}_{w} A$ that contain $Q$.

Proof. By Proposition 2.3, $\operatorname{spec}_{w} A$ is a link-closed subset of spec $A$. By Assumptions 4 and $5, \operatorname{spec}_{w} A$ is invariant under $\mathcal{H}$. In view of Lemma 2.4 and the fact that localization preserves links, we see that for any $K \in \operatorname{spec}_{w} A$, the clique of $K / J_{w}$ in $\operatorname{spec} A / J_{w}$ is a singleton. Therefore the theorem follows from Corollary 2.10 .

\section{Homogeneity of Cliques}

We continue with the setup and assumptions of (1.1), and we impose Assumption 7 throughout. Here we globalize the results of the previous section, by proving that cliques in spec $A$ have the form of orbits with respect to certain subgroups of $\mathcal{H}$. Further, for each $w \in \mathcal{W}$ there is a subgroup $T_{w} \subseteq \mathcal{H}$ that determines all the cliques within $\operatorname{spec}_{w} A$, as well as a finite subset of $T_{w}$ that determines almost all the links within $\operatorname{spec}_{w} A$.

3.1. For $P \in \operatorname{spec} A$, define

$$
\begin{aligned}
T_{P} & =\{\tau \in \mathcal{H} \mid \tau(P) \in \operatorname{clique}(P)\}, \\
\Sigma_{P} & =\{\sigma \in \mathcal{H} \mid \sigma(P) \rightsquigarrow P\}, \\
N_{P} & =\{\eta \in \mathcal{H} \mid \eta(P)=P\} .
\end{aligned}
$$

The following proposition exhibits some general observations relating these sets of automorphisms to the structure of the clique of $P$.

Proposition. (a) $T_{P}$ is a subgroup of $\mathcal{H}$, and the $T_{P}$-orbit of $P$ coincides with its clique.

(b) $N_{P}$ is a subgroup of $T_{P}$, and there is a bijection $T_{P} / N_{P} \rightarrow$ clique $(P)$ given by the rule $\tau N_{P} \mapsto \tau(P)$.

(c) If $T$ is a subgroup of $T_{P}$ such that all primes linked to $P$ lie in the $T$-orbit of $P$, then the clique of $P$ equals its $T$-orbit.

(d) If $P$ is not a minimal prime, then $N_{P} \subseteq \Sigma_{P}$ and $\Sigma_{P}$ generates $T_{P}$.

Proof. We have $P \in \operatorname{spec}_{w} A$ for some $w \in \mathcal{W}$. By Proposition 2.3 and Theorem 2.11, clique $(P) \subseteq \operatorname{spec}_{w} A$ and whenever $Q \rightsquigarrow Q^{\prime}$ in $\operatorname{spec}_{w} A$ there exists $\sigma \in \mathcal{H}$ such that $Q=\sigma\left(Q^{\prime}\right)$. It follows that the clique of $P$ is contained in its $\mathcal{H}$-orbit. Hence, clique $(P)=\left\{\tau(P) \mid \tau \in T_{P}\right\}$.

(a) First observe that clique $(P)$ is invariant under every $\tau \in T_{P}$ : since automorphisms preserve links, $\tau$ maps clique $(P)$ into clique $(\tau(P))$, and the latter coincides with clique $(P)$ because $\tau \in T_{P}$. It follows that $T_{P}$ is closed under composition. Further, for $\tau \in T_{P}$ we have $P \in \operatorname{clique}\left(\tau^{-1}(P)\right)$ and so $\tau^{-1}(P) \in \operatorname{clique}(P)$, whence $\tau^{-1} \in T_{P}$. Therefore $T_{P}$ is a subgroup of $\mathcal{H}$. 
(b) This is clear.

(c) Obviously the $T$-orbit of $P$ is contained in its clique. Given $Q \in \operatorname{clique}(P)$, choose primes $P_{0}=P, P_{1}, \ldots, P_{m}=Q$ such that for $l=1, \ldots, m$ either $P_{l-1} \rightsquigarrow P_{l}$ or $P_{l} \rightsquigarrow P_{l-1}$. If $P_{l-1} \rightsquigarrow P_{l}$, choose $\tau_{l} \in T_{P}$ such that $\tau_{l}(P)=P_{l}$ and note that $\tau_{l}^{-1}\left(P_{l-1}\right) \rightsquigarrow P$. Then $\tau_{l}^{-1}\left(P_{l-1}\right)=\sigma_{l}(P)$ for some $\sigma_{l} \in T$, and $P_{l-1}=\sigma_{l}\left(P_{l}\right)$ (because $\sigma_{l}$ and $\tau_{l}$ commute). Similarly, if $P_{l} \rightsquigarrow P_{l-1}$, then $P_{l}=\sigma_{l}\left(P_{l-1}\right)$ for some $\sigma_{l} \in T$. Hence, $Q=\sigma(P)$ for some automorphism $\sigma$ of the form $\sigma_{m}^{ \pm 1} \sigma_{m-1}^{ \pm 1} \cdots \sigma_{1}^{ \pm 1}$, and therefore $Q$ lies in the $T$-orbit of $P$.

(d) It follows from Theorem 2.6 that $N_{P} \subseteq \Sigma_{P}$. Let $T$ be the subgroup of $T_{P}$ generated by $\Sigma_{P}$. By part (c), the $T$-orbit of $P$ equals its clique. Given $\tau \in T_{P}$, we have $\tau(P)=\eta(P)$ for some $\eta \in T$. Then $\eta^{-1} \tau(P)=P$ and so $\eta^{-1} \tau \in N_{P} \subseteq \Sigma_{P}$, whence $\tau \in T$. Therefore $T_{P}=T$.

3.2. Lemma. Let $P, P^{\prime} \in \operatorname{prim}_{w} A$ for some $w \in \mathcal{W}$. Then $T_{P^{\prime}}=T_{P}, \Sigma_{P^{\prime}}=\Sigma_{P}$, and $N_{P^{\prime}}=N_{P}$.

Proof. By Assumption 7, $P^{\prime}=\rho(P)$ for some $\rho \in \mathcal{H}$. Since $\mathcal{H}$ is abelian, it follows that

$$
\begin{aligned}
T_{P^{\prime}} & =\{\tau \in \mathcal{H} \mid \tau \rho(P) \in \operatorname{clique}(\rho(P))\}=\{\tau \in \mathcal{H} \mid \rho \tau(P) \in \rho(\operatorname{clique}(P))\} \\
& =\{\tau \in \mathcal{H} \mid \tau(P) \in \operatorname{clique}(P)\}=T_{P} .
\end{aligned}
$$

Similarly, $\Sigma_{P^{\prime}}=\Sigma_{P}$ and $N_{P^{\prime}}=N_{P}$.

3.3. Let $w \in \mathcal{W}$, choose $P_{w} \in \operatorname{prim}_{w} A$, and set

$$
T_{w}=T_{P_{w}} ; \quad \Sigma_{w}=\Sigma_{P_{w}} ; \quad N_{w}=N_{P_{w}} .
$$

By Lemma 3.2, these definitions are independent of the choice of $P_{w}$. We write

$$
\Sigma_{w} / N_{w}=\left\{\sigma N_{w} \mid \sigma \in \Sigma_{w}\right\}
$$

for the image of $\Sigma_{w}$ in the group $T_{w} / N_{w}$. In view of Theorem 2.11, the finite set

$$
\Sigma_{w} \cap\left\{h_{w, 1}, \ldots, h_{w, m(w)}\right\}
$$

contains a full set of coset representatives for $\Sigma_{w} / N_{w}$.

Theorem. (a) The group $T_{w}$ determines all the cliques within $\operatorname{spec}_{w}$ A: the clique of each prime $P \in \operatorname{spec}_{w} A$ coincides with its $T_{w}$-orbit. Moreover, $N_{w} \subseteq N_{P}$, and so there is a surjection $T_{w} / N_{w} \rightarrow$ clique $(P)$ given by the rule $\tau N_{w} \mapsto \tau(P)$.

(b) The finite set $\Sigma_{w} / N_{w}$ determines almost all the links within $\operatorname{spec}_{w} A$ : if $\left\{\sigma_{1}, \ldots, \sigma_{r}\right\}$ is a transversal for $\Sigma_{w} / N_{w}$, then

$$
\left\{\sigma_{1}(P), \ldots, \sigma_{r}(P)\right\}=\left\{Q \in \operatorname{spec}_{w} A \mid Q \rightsquigarrow P\right\}
$$

for $P \in \operatorname{spec}_{w} A$, except possibly when $P$ is a minimal prime of $A$. (In that case, clique $(P)=\{P\}$, but $P$ might not be linked to itself.)

(c) Any transversal for $\Sigma_{w} / N_{w}$ generates $T_{P}$ modulo $N_{P}$ for each $P \in \operatorname{spec}_{w} A$. In particular, $T_{w} \subseteq T_{P}$ and $T_{P} / N_{P}$ is a homomorphic image of $T_{w} / N_{w}$.

Proof. First consider a prime $P \in \operatorname{spec}_{w} A$ which happens to be a minimal prime of $A$. Then $P$ must also be minimal in $\operatorname{spec}_{w} A$, and so $P=J_{w}$. In this case, 
$P$ is $\mathcal{H}$-invariant (Assumption 3 ), and so $T_{P}=N_{P}=\mathcal{H}$. On the other hand, clique $(P)=\{P\}$ by Proposition 2.3 and Theorem 2.11. Hence, statements (a), (b), and (c) are trivially satisfied for this $P$.

Now fix a prime $P \in \operatorname{spec}_{w} A$ which is not a minimal prime of $A$. We first show that $N_{w} \subseteq N_{P}$. By Proposition 1.3, $P=\bigcap_{\lambda \in \Lambda} P_{\lambda}$ for some primitive ideals $P_{\lambda} \in \operatorname{prim}_{w} A$. Since the automorphisms in $N_{w}$ stabilize each $P_{\lambda}$, they must also stabilize $P$; thus $N_{w} \subseteq N_{P}$.

Next, we show that $\Sigma_{w} \subseteq \Sigma_{P}$. It follows from Lemma 2.4 that the clique of each $P_{\lambda} / J_{w}$ in spec $A / J_{w}$ is a singleton. Hence, the $P_{\lambda} / J_{w}$ are localizable primes of $A / J_{w}[\mathbf{2 0}, 7.3 .1 ; \mathbf{3 1}, 4.3 .16 ; \mathbf{1 3}, 12.21]$. Now for any $\tau \in \Sigma_{w}$, we have $\tau\left(P_{\lambda}\right) \rightsquigarrow P_{\lambda}$ for all $\lambda$. Thus the hypotheses of $[\mathbf{2}, 4.2]$ are satisfied, and so either $\tau(P)=P$ or $\tau(P) \rightsquigarrow P$. Since $P \rightsquigarrow P$ (Theorem 2.6), this shows that $\tau \in \Sigma_{P}$, as claimed.

(b) Each $\sigma_{i} \in \Sigma_{w} \subseteq \Sigma_{P}$, and so $\sigma_{i}(P) \rightsquigarrow P$ for all $i=1, \ldots, r$. Conversely, let $Q \in \operatorname{spec}_{w} A$ such that $Q \rightsquigarrow P$. If $Q=P$, write $Q=\sigma(P)$ where $\sigma=$ id $\in \mathcal{H}$. If $Q \neq P$, then by Theorem 2.11, there exists $\sigma \in \mathcal{H}$ such that $Q=\sigma(P)$ and $\sigma\left(P_{\lambda}\right) \rightsquigarrow P_{\lambda}$ for all $\lambda$. In either case, $\sigma \in \Sigma_{w}$, whence $\sigma \in \sigma_{i} N_{w} \subseteq \sigma_{i} N_{P}$ for some $i$, and so $Q=\sigma_{i}(P)$.

(c) Since $P$ is non-minimal, $\Sigma_{P}$ generates $T_{P}$ by Proposition 3.1(d). Let $\left\{\sigma_{1}, \ldots\right.$, $\left.\sigma_{r}\right\}$ be a transversal for $\Sigma_{w} / N_{w}$. In view of part (b), $\Sigma_{P} \subseteq \sigma_{1} N_{P} \cup \cdots \cup \sigma_{r} N_{P}$, and therefore the cosets $\sigma_{1} N_{P}, \ldots, \sigma_{r} N_{P}$ generate $T_{P} / N_{P}$.

(a) We have seen that $\Sigma_{w} \subseteq \Sigma_{P}$. Since $T_{w}$ is generated by $\Sigma_{w}$ (Proposition 3.1(d)), we thus have $T_{w} \subseteq T_{P}$. On the other hand, all primes linked to $P$ lie in the $T_{w}$-orbit of $P$ by part (b). Therefore Proposition 3.1(c) shows that the clique of $P$ equals its $T_{w}$-orbit. The final statement of part (a) is clear.

3.4. Theorem. Let $w \in \mathcal{W}$ and $P \in \operatorname{spec}_{w} A$. Assume that there is a subgroup $\mathcal{H}_{w}$ of $\mathcal{H}$ such that

(a) $J_{w}$ is $\mathcal{H}_{w}$-polynormal;

(b) For all $h \in \mathcal{H}_{w}$, the $\langle h\rangle$-orbit of $P$ is either infinite or a singleton.

Then $T_{P} / N_{P}$ is a free abelian group of finite rank. Consequently, clique $(P)$ is either infinite or a singleton.

Proof. We may choose the automorphisms $h_{w, j}$ in the statement of Theorem 2.11 from the group $\mathcal{H}_{w}$. Let $T$ be the subgroup of $\mathcal{H}_{w}$ generated by the set

$$
\left\{h_{w, j} \mid j \in\{1, \ldots, m(w)\} \text { and } h_{j, w}(P) \rightsquigarrow P\right\} .
$$

In view of Proposition 2.3 and Theorem 2.11, all primes linked to $P$ occur in the $T$-orbit of $P$. Thus by Proposition 3.1(c), the clique of $P$ equals its $T$-orbit. Hence, $T_{P}=T N_{P}$, and so $T_{P} / N_{P} \cong T /\left(T \cap N_{P}\right)$. Hypothesis (b) shows that $T /\left(T \cap N_{P}\right)$ is torsionfree. Since $T$ is finitely generated, we conclude that $T /\left(T \cap N_{P}\right)$ is free abelian of finite rank.

The final statement of the theorem follows from the existence of a bijection from $T_{P} / N_{P}$ onto clique $(P)$ (Proposition 3.1(b)).

3.5. Theorem. The intersection of the clique of any prime $P \in \operatorname{spec} A$ is a localizable semiprime ideal. Further, if $P \in \operatorname{spec}_{w} A$ and the conditions of Theorem 3.4 hold, this intersection is prime and lies in $\operatorname{spec}_{w} A$.

Proof. Set $J_{P}=\bigcap$ clique $(P)$. Now clique $(P) \subseteq \operatorname{spec}_{w} A$ by Proposition 2.3, so $J_{w} \subseteq J_{P}$. By Theorem 3.3(a), the clique of $P$ coincides with its $T_{w}$-orbit, and therefore $J_{P}=\left(P: T_{w}\right)$. Hence, $J_{P}$ is a $T_{w}$-prime ideal. 
Let $Q_{1}, \ldots, Q_{m}$ be the primes of $A$ minimal over $J_{P}$. Each $Q_{j}$ contains $J_{w}$, and the $Q_{j}$ form a single $T_{w}$-orbit (as explained in (1.8)). At least one of the $Q_{j}$ is contained in $P$, say $Q_{1} \subseteq P$; thus $Q_{1} \in \operatorname{spec}_{w} A$. Now Theorem 3.3(a) shows that the clique of $Q_{1}$ coincides with its $T_{w}$-orbit, and so

$$
\operatorname{clique}\left(Q_{1}\right)=\left\{Q_{1}, \ldots, Q_{m}\right\}
$$

In particular, the set $\left\{Q_{1}, \ldots, Q_{m}\right\}$ is link-closed, and therefore $J_{P}$ is localizable $[\mathbf{2 0}, 7.3 .1 ; \mathbf{1 3}, 12.21]$.

If the conditions of Theorem 3.4 hold, then since the clique of $Q_{1}$ is finite, Theorem 3.4 implies that $m=1$. Therefore $J_{P}=Q_{1}$ is a prime ideal in $\operatorname{spec}_{w} A$ in this case.

3.6. In the situation of Theorems 3.4 and 3.5 , spec $A$ can be parametrized by the set of localizable primes. Namely, $\operatorname{spec} A$ is the disjoint union of the sets

$$
V_{Q}=\{P \in \operatorname{spec} A \mid \bigcap \operatorname{clique}(P)=Q\}
$$

as $Q$ ranges over the localizable primes. This provides a finer partition of $\operatorname{spec} A$ than that given by the sets $\operatorname{spec}_{w} A$, since each $\operatorname{spec}_{w} A$ is the union of the sets $V_{Q}$ for localizable primes $Q \in \operatorname{spec}_{w} A$, and in the typical examples there are infinitely many such $Q$.

3.7. It is well known that cliques are invariant under the automorphisms associated with regular normal elements. We record a convenient form of this fact as follows.

Lemma. Let $R$ be a noetherian ring satisfying the second layer condition, $I$ an ideal of $R$, and $c$ an element of $R$ which is regular and $\alpha$-normal modulo $I$ for some $\alpha \in$ Aut $R$. If $P$ is a prime ideal of $R$ that contains $I$, then $\alpha(P)$ and $\alpha^{-1}(P)$ lie in the clique of $P$. Further, if $c \notin P$, then $\alpha(P)=P$.

Proof. Note that $\alpha(I) c \subseteq c I+I=I$, whence $\alpha(I) \subseteq I$. Similarly, $\alpha^{-1}(I) \subseteq I$, and so $\alpha(I)=I$. Hence, after passing to $R / I$ there is no loss of generality in assuming that $I=0$. Now $c \alpha^{-1}(P)=P c \subseteq P$ and $\alpha(P) c=c P \subseteq P$. If $c \notin P$, it follows that $\alpha^{ \pm 1}(P) \subseteq P$, and thus $\alpha(P)=P$.

Since $c$ is regular in $R$, we have $c R / c P=R c / \alpha(P) c \cong R / \alpha(P)$ as left $R$-modules and $c R / c P \cong R / P$ as right $R$-modules. Hence, $c R / c P$ gives a bond from $R / \alpha(P)$ to $R / P$, and therefore $\alpha(P) \in \operatorname{clique}(P)$ by $[\mathbf{2 0}, 8.2 .4]$ (cf. Theorem 2.8 above). It follows that $P \in \operatorname{clique}\left(\alpha^{-1}(P)\right)$, and thus $\alpha^{-1}(P) \in \operatorname{clique}(P)$.

3.8. Recall that a sequence $x_{1}, \ldots, x_{m}$ of elements in a ring $R$ is called a regular sequence if $x_{1}$ is a regular element of $R$ and $x_{j}$ is regular modulo $\left\langle x_{1}, \ldots, x_{j-1}\right\rangle$ for $j=2, \ldots, m$.

Let $w \in \mathcal{W}$, and let $x_{w, 1}, \ldots, x_{w, m(w)}$ be an $\mathcal{H}$-polynormal sequence of generators for $J_{w}$, with corresponding automorphisms $h_{w, j} \in \mathcal{H}$, as in Theorem 2.11.

Theorem. Assume that $x_{w, 1}, \ldots, x_{w, m(w)}$ is a regular sequence. Then $h_{w, 1}, \ldots$, $h_{w, m(w)}$ all lie in $T_{w}$, and so $T_{w}=\left\langle h_{w, 1}, \ldots, h_{w, m(w)}\right\rangle N_{w}$. Hence, the clique of each prime $P \in \operatorname{spec}_{w} A$ coincides with its $\left\langle h_{w, 1}, \ldots, h_{w, m(w)}\right\rangle$-orbit.

Proof. Since $x_{w, j} \in J_{w} \subseteq P_{w}$ for all $j$, Lemma 3.7 implies that $h_{w, j}\left(P_{w}\right) \in$ clique $\left(P_{w}\right)$ for all $j$, that is, $h_{w, j} \in T_{w}$. The remainder of the theorem now follows from Theorem 3.3. 


\section{Quantum Affine Spaces}

Multiparameter quantum coordinate rings of affine space afford a straightforward illustration of the axiom system and results of Sections 1-3. Some results in the direction followed below were obtained by Lenagan and the second author in connection with [11, Section 2].

4.1. Let $\mathbf{q}=\left(q_{i j}\right)$ be a multiplicatively antisymmetric $n \times n$ matrix over a field $\mathbb{K}$, that is, $q_{i i}=1$ and $q_{j i}=q_{i j}^{-1}$ for all $i, j$. Let $A=\mathcal{O}_{\mathbf{q}}\left(\mathbb{K}^{n}\right)$ be the $\mathbb{K}$-algebra generated by elements $x_{1}, \ldots, x_{n}$ subject only to the relations $x_{i} x_{j}=q_{i j} x_{j} x_{i}$ for $i, j=1, \ldots, n$; this algebra is the general multiparameter quantum coordinate ring of affine $n$-space over $\mathbb{K}$. Suppose that the multiplicative subgroup of $\mathbb{K}^{\times}$generated by the $q_{i j}$ is torsionfree. We shall show that under these conditions, $A$ fits the setup and assumptions of (1.1). If $\mathbb{K}$ is algebraically closed, Assumption 7 holds as well.

4.2. It is well known that $A$ is a noetherian domain; in fact, $A$ can easily be expressed as an iterated skew polynomial ring of the form

$$
\mathbb{K}\left[x_{1}\right]\left[x_{2} ; \sigma_{2}\right] \cdots\left[x_{n} ; \sigma_{n}\right] .
$$

For any $n$-tuple $\alpha \in\left(\mathbb{K}^{\times}\right)^{n}$, there is a $\mathbb{K}$-algebra automorphism $h_{\alpha}$ of $A$ such that $h_{\alpha}\left(x_{i}\right)=\alpha_{i} x_{i}$ for all $i$. The set $\mathcal{H}=\left\{h_{\alpha} \mid \alpha \in\left(\mathbb{K}^{\times}\right)^{n}\right\}$ is then an abelian subgroup of Aut $A$, isomorphic to the torus $\left(\mathbb{K}^{\times}\right)^{n}$. Let $\mathcal{W}$ be the set of subsets of $\{1, \ldots, n\}$, and for $w \in \mathcal{W}$ let $\operatorname{spec}_{w} A$ be the set of those primes $P \in \operatorname{spec} A$ such that

$$
P \cap\left\{x_{1}, \ldots, x_{n}\right\}=\left\{x_{i} \mid i \in w\right\} .
$$

Then $\operatorname{spec} A$ is the disjoint union of the $\operatorname{sets} \operatorname{spec}_{w} A$.

Assumptions 2-5 are easy to verify. For each $w \in \mathcal{W}$, the ideal $J_{w}=\left\langle x_{i} \mid i \in w\right\rangle$ is the minimum element of $\operatorname{spec}_{w} A$, and $J_{w}$ is obviously $\mathcal{H}$-invariant. Since all the $x_{i}$ are $\mathcal{H}$-normal, $J_{w}$ is $\mathcal{H}$-polynormal. Let $E_{w}$ denote the multiplicative set in $A / J_{w}$ generated by the images of those $x_{j}$ with $j \notin w$. These generators are normal $\mathcal{H}$-eigenvectors, and since they are normal, the primes in $A / J_{w}$ which are disjoint from $E_{w}$ are precisely the primes which do not contain $x_{j}+J_{w}$ for any $j \notin w$. These are exactly the primes of the form $P / J_{w}$ for $P \in \operatorname{spec}_{w} A$.

To check Assumption 1, note that since $A$ is an iterated normalizing extension of $\mathbb{K}$ (each $x_{i}$ normalizes the subalgebra $\mathbb{K}\left\langle x_{1}, \ldots, x_{i-1}\right\rangle$ ), it is a constructible $\mathbb{K}$ algebra in the terminology of $[\mathbf{3 1}, 9.4 .12]$. Thus by $[\mathbf{3 1}, 9.4 .21], A$ is Jacobson; in fact, $A$ satisfies the Nullstellensatz.

4.3. Verification of Assumptions 6 and 7 requires some analysis of the localizations $A_{w}$. Observe that any $A_{w}$ is generated by the elements $\left(x_{j}+J_{w}\right)^{ \pm 1}$ for $j \notin w$, subject to our initial relations. Note also that the induced action of $\mathcal{H}$ on $A_{w}$ consists of the $\mathbb{K}$-algebra automorphisms sending $x_{j}+J_{w}$ to $\beta_{j} x_{j}+J_{w}$ for any choices of scalars $\beta_{j} \in \mathbb{K}^{\times}, j \notin w$.

The algebra $A_{w}$ can be viewed as one of the algebras $P(\boldsymbol{\lambda})$ investigated in $[\mathbf{3 0}]$. Here $\boldsymbol{\lambda}=\left(\lambda_{i j}\right)$ is a multiplicatively antisymmetric $m \times m$ matrix over $\mathbb{K}$, and $P(\boldsymbol{\lambda})$ is the $\mathbb{K}$-algebra generated by elements $y_{1}^{ \pm 1}, \ldots, y_{m}^{ \pm 1}$ subject only to the relations $y_{i} y_{j}=\lambda_{i j} y_{j} y_{i}$ for all $i, j$. In particular, $P(\boldsymbol{\lambda})$ is isomorphic to a localization of $\mathcal{O}_{\boldsymbol{\lambda}}\left(\mathbb{K}^{m}\right)$, and there is a torus $H=\left\{h_{\alpha} \mid \alpha \in\left(\mathbb{K}^{\times}\right)^{m}\right\}$ of automorphisms of $P(\boldsymbol{\lambda})$ just as in (4.2), where $h_{\alpha}\left(y_{i}\right)=\alpha_{i} y_{i}$ for all $i$. It will be convenient to use the standard multi-index notation for monomials in $P(\boldsymbol{\lambda})$ : thus $y^{s}=y_{1}^{s_{1}} y_{2}^{s_{2}} \cdots y_{m}^{s_{m}}$ for any $m$-tuple $s=\left(s_{1}, \ldots, s_{m}\right) \in \mathbb{Z}^{m}$. 
4.4. Theorem. Let $R=P(\boldsymbol{\lambda})$ as defined in (4.3), and assume that the multiplicative subgroup of $\mathbb{K}^{\times}$generated by the $\lambda_{i j}$ is torsionfree.

(a) All ideals of $R$ are centrally generated. In fact, extension and contraction give inverse isomorphisms between the lattices of ideals of $Z(R)$ and $R$.

(b) All primitive ideals of $R$ are maximal.

(c) If $\mathbb{K}$ is algebraically closed, the group $H$ acts transitively on $\max R$.

Proof. Let $S$ denote the following subgroup of $\mathbb{Z}^{m}$ :

$$
S=\left\{s \in \mathbb{Z}^{m} \mid \lambda_{1 j}^{s_{1}} \lambda_{2 j}^{s_{2}} \cdots \lambda_{m j}^{s_{m}}=1 \text { for all } j=1, \ldots, m\right\}=\left\{s \in \mathbb{Z}^{m} \mid y^{s} \in Z(R)\right\} .
$$

Since the multiplicative group $\left\langle\lambda_{i j}\right\rangle$ is torsionfree, so is the additive group $\mathbb{Z}^{m} / S$. Hence, there exists a basis $s_{1}, \ldots, s_{m}$ for $\mathbb{Z}^{m}$ such that for some $t \in\{0, \ldots, m\}$, the sequence $s_{t+1}, \ldots, s_{m}$ is a basis for $S$. The monomials $z_{i}=y^{s_{i}}$ and their inverses form a new set of generators for $R$, satisfying the relations $z_{i} z_{j}=\mu_{i j} z_{j} z_{i}$ for all $i$, where

$$
\mu_{i j}=\prod_{k, l=1}^{m} \lambda_{k l}^{s_{i k} s_{j l}}
$$

Thus, we obtain a presentation of $R$ in the form $P(\boldsymbol{\mu})$, where $\boldsymbol{\mu}=\left(\mu_{i j}\right)$. Note that the multiplicative subgroup of $\mathbb{K}^{\times}$generated by the $\mu_{i j}$ is torsionfree.

The automorphisms in $H$ have the form $h_{\alpha}$ given in (4.3), and

$$
h_{\alpha}\left(z_{i}\right)=\left(\prod_{j=1}^{m} \alpha_{j}^{s_{i j}}\right) z_{i},
$$

for all $\alpha, i$. Thus $h_{\alpha}$ can be described as a $\mathbb{K}$-algebra automorphism of the form $k_{\beta}$, where $\beta \in\left(\mathbb{K}^{\times}\right)^{m}$ and $k_{\beta}\left(z_{i}\right)=\beta_{i} z_{i}$ for all $i$. Conversely, since $\left(s_{i j}\right) \in G L_{m}(\mathbb{Z})$, every $k_{\beta}$ can be expressed as an $h_{\alpha}$. Thus $H=\left\{k_{\beta} \mid \beta \in\left(\mathbb{K}^{\times}\right)^{m}\right\}$.

Therefore we may assume, without loss of generality, that $s_{1}, \ldots, s_{m}$ is the standard basis for $\mathbb{Z}^{m}$, that is, $y^{s_{i}}=y_{i}$ for all $i$. Hence, $y_{t+1}, \ldots, y_{m} \in Z(R)$, and no monomial in $y_{1}, \ldots, y_{t}$ is central except in the trivial case where all exponents are zero.

Let $R_{t}$ and $R_{t}^{\prime}$ denote the subalgebras of $R$ generated by $y_{1}^{ \pm 1}, \ldots, y_{t}^{ \pm 1}$ and $y_{t+1}^{ \pm 1}, \ldots, y_{m}^{ \pm 1}$ respectively. We claim that $R_{t}$ is a central simple $\mathbb{K}$-algebra, and that $R_{t}^{\prime}=Z(R)$. To establish the first claim, it suffices, by [30, 1.3], to show that for any nonzero $t$-tuple $r \in \mathbb{Z}^{t}$, there is an index $j \in\{1, \ldots, t\}$ such that $\lambda_{1 j}^{r_{1}} \lambda_{2 j}^{r_{2}} \cdots \lambda_{t j}^{r_{t}} \neq 1$. This is immediate from the definition of $S$. Therefore $R_{t}$ is a central simple $\mathbb{K}$-algebra, as claimed.

Observe that the multiplication map $R_{t} \otimes_{\mathbb{K}} R_{t}^{\prime} \rightarrow R$ is a $\mathbb{K}$-algebra isomorphism. Since $R_{t}$ is central simple while $R_{t}^{\prime}$ is commutative, the center of $R_{t} \otimes_{\mathbb{K}} R_{t}^{\prime}$ must be $1 \otimes R_{t}^{\prime}$. Hence, $R_{t}^{\prime}$ equals the center of $R$, as claimed.

(a) This follows from the tensor product decomposition of $R$ just given, together with the standard analysis of ideals in tensor products with one central simple factor (e.g., [19, V.6.1, p. 109]).

(b) Note that $Z(R)=R_{t}^{\prime}=\mathbb{K}\left[y_{t+1}^{ \pm 1}, \ldots, y_{m}^{ \pm 1}\right]$ is a commutative Jacobson ring. Further, if we view $R$ as constructed from $Z(R)$ by successively adjoining $y_{1}^{ \pm 1}, \ldots$, $y_{t}^{ \pm 1}$, then $R$ is obtained from $Z(R)$ by an iterated sequence of normalizing extensions. Hence, $R$ is a constructible $Z(R)$-algebra, and thus by [31, 9.4.21], all 
primitive ideals of $R$ contract to maximal ideals of $Z(R)$. In view of the lattice isomorphism established in part (a), it follows that all primitive ideals of $R$ are maximal.

(c) If $\mathbb{K}$ is algebraically closed, the maximal ideals of $Z(R)$ are the ideals of the form $\left\langle y_{t+1}-\gamma_{t+1}, \ldots, y_{m}-\gamma_{m}\right\rangle$ for $\gamma_{t+1}, \ldots, \gamma_{m} \in \mathbb{K}^{\times}$. Clearly the restriction of $H$ to $Z(R)$ permutes these ideals transitively. In view of part (a), it follows that $H$ also permutes $\max R$ transitively.

4.5. In view of (4.3), Theorem 4.4 verifies that Assumption 6 is satisfied. We now impose the condition that $\mathbb{K}$ is algebraically closed; then Theorem 4.4 together with Corollary 1.4 verifies Assumption 7. This completes the check of Assumptions 1-7 for the algebra $A=\mathcal{O}_{\mathbf{q}}\left(\mathbb{K}^{n}\right)$, under our hypotheses that $\mathbb{K}$ is algebraically closed and the multiplicative group $\left\langle q_{i j}\right\rangle$ is torsionfree. Hence, all the results of Sections 1-3 are applicable to $A$. One consequence is that $\operatorname{spec} A$ has normal separation (whether or not $\mathbb{K}$ is algebraically closed - see Proposition 1.7); this has been noted in $[\mathbf{1 1}, 2.4]$.

In particular, we can find lattices $\mathcal{H}_{w} \subseteq \mathcal{H}$ which determine the cliques in $\operatorname{spec}_{w} A$, as follows. For $i=1, \ldots, n$, let $\tau_{i}$ denote the automorphism $h_{\left(q_{i 1}, \ldots, q_{i n}\right)}$ in $\mathcal{H}$; thus $\tau_{i}\left(x_{j}\right)=q_{i j} x_{j}$ for all $j$, and $x_{i}$ is $\tau_{i}$-normal in $A$. For $w \in \mathcal{W}$, set $\mathcal{H}_{w}=\left\langle\tau_{j} \mid j \in w\right\rangle$ and observe that the $x_{j}$ for $j \in w$ (taken in any order) form an $\mathcal{H}_{w}$-polynormal regular sequence of generators for $J_{w}$. Thus by Theorem 3.8, the group $T_{w}$ of (3.3) has the form $\mathcal{H}_{w} N_{w}$, and the clique of each prime in $\operatorname{spec}_{w} A$ coincides with its $\mathcal{H}_{w}$-orbit.

Further, Theorem 3.4 applies in this situation, as a result of the following easy lemma. It is presumably well known, but we have not located a reference in the literature. One form of the idea is used in $[\mathbf{2 2}, 7.17]$.

4.6. Lemma. Let $R$ be a $\mathbb{K}$-vector space and $\tau$ a $\mathbb{K}$-linear automorphism of $R$. Suppose that $R$ is the sum of its $\tau$-eigenspaces, and that the multiplicative subgroup of $\mathbb{K}^{\times}$generated by the eigenvalues of $\tau$ is torsionfree. Then any subspace $I$ of $R$ whose $\langle\tau\rangle$-orbit is finite must be $\tau$-invariant.

Proof. By hypothesis, $\tau^{m}(I)=I$ for some integer $m \geq 1$. As $R$ is spanned by $\tau^{m}$ eigenvectors, $I$ must be spanned by $\tau^{m}$-eigenvectors. Hence, it suffices to show that every nonzero $\tau^{m}$-eigenvector $x \in R$ is also a $\tau$-eigenvector. Write $x=x_{1}+\cdots+x_{t}$ where the $x_{i}$ are nonzero $\tau$-eigenvectors with distinct eigenvalues $\lambda_{1}, \ldots, \lambda_{t}$. Then $\lambda_{1}^{m} x_{1}+\cdots+\lambda_{t}^{m} x_{t}=\tau^{m}(x)=\lambda x$ for some $\lambda \in \mathbb{K}$. Since eigenvectors with distinct eigenvalues are linearly independent, $\lambda_{i}^{m}=\lambda$ for all $i$. Consequently, because the $\lambda_{i}$ lie in a torsionfree subgroup of $\mathbb{K}^{\times}$, they must all coincide. Therefore $t=1$ and $x$ is a $\tau$-eigenvector, as desired.

4.7. Theorem. Let $w \in \mathcal{W}$.

(a) The group $\mathcal{H}_{w}$ is a lattice of rank at most card $w$.

(b) The clique of each prime in $\operatorname{spec}_{w} A$ coincides with its $\mathcal{H}_{w}$-orbit.

(c) Each clique in $\operatorname{spec}_{w} A$ is either infinite or a singleton.

Proof. Part (b) has already been noted in (4.5).

(a) Any automorphism in $\mathcal{H}_{w}$ has the form $h_{\alpha}$ where the entries of $\alpha$ lie in the multiplicative group $\left\langle q_{i j}\right\rangle$. Since the latter group is assumed to be torsionfree, we see that $h_{\alpha}$ cannot have finite order unless it is the identity. Therefore $\mathcal{H}_{w}$ is 
torsionfree. Since it is generated by card $w$ automorphisms, it is thus a free abelian group of rank at most card $w$.

(c) Given $h_{\alpha} \in \mathcal{H}_{w}$ as above, observe that $A$ is the sum of its $h_{\alpha}$-subspaces, and that the eigenvalues of $h_{\alpha}$ all lie in the torsionfree group $\left\langle q_{i j}\right\rangle$. Hence, Lemma 4.6 shows that any ideal of $A$ whose $\left\langle h_{\alpha}\right\rangle$-orbit is finite must be $h_{\alpha}$-invariant. Thus, by Theorem 3.4, the clique of any prime in $\operatorname{spec}_{w} A$ is either infinite or a singleton.

4.8. In our present case, it is easy to determine the links in $\operatorname{spec} A$. Given $w \in \mathcal{W}$ and $P \in \operatorname{spec}_{w} A$, we know that $P \rightsquigarrow P$ unless $P=0$ (Theorem 2.6), and that the only possible other primes linked to $P$ are the primes $\tau_{j}(P)$ for $j \in w$ (Proposition 2.3 and Theorem 2.11). In fact, $\tau_{j}(P) \rightsquigarrow P$ for all $j \in w$. To see this, fix $j \in w$, set $J=\left\langle x_{i} \mid i \in w \backslash\{j\}\right\rangle$, and observe that $x_{j}$ is regular modulo $J$. Since $J_{w}=\left\langle x_{j}\right\rangle+J$ and $x_{j}$ is $\tau_{j}$-normal, it follows that there is a bimodule subfactor of $J_{w} / J_{w}^{2}$ which is a bond from $A / \tau_{j}(P)$ to $A / P$. On the other hand, the cliques of $\tau_{j}(P) / J_{w}$ and $P / J_{w}$ in spec $A / J_{w}$ are singletons (because of Lemma 2.4), and so Lemma 2.9 shows that $\tau_{j}(P) \rightsquigarrow P$. To summarize:

Theorem. Let $w \in \mathcal{W}$, and let $P$ be a nonzero prime in $\operatorname{spec}_{w} A$. Then

$$
\{Q \in \operatorname{spec} A \mid Q \rightsquigarrow P\}=\{P\} \cup\left\{\tau_{j}(P) \mid j \in w\right\} .
$$

4.9. Example. The standard one-parameter coordinate ring of quantum affine space is often denoted $\mathcal{O}_{q}\left(\mathbb{K}^{n}\right)$, where $q \in \mathbb{K}^{\times}$. This algebra is just the case of $A=\mathcal{O}_{\mathbf{q}}\left(\mathbb{K}^{n}\right)$ where $q_{i j}=q$ for all $i<j$. Suppose that $q \neq 1$ and that $q$ is not a root of unity.

As observed in [37, pp. 144-145], it follows from [30, 1.3] that the localization $A_{w}$ is simple if and only if $n-$ card $w$ is even. In that case, $\operatorname{spec}_{w} A=\left\{J_{w}\right\}$.

Now suppose that $m=n-\operatorname{card} w$ is odd, and let $l_{1}<\cdots<l_{m}$ be a list of the elements of $\{1, \ldots, n\} \backslash w$ in ascending order. Set $y_{i}=x_{l_{i}}+J_{w}$ for $i=1, \ldots, m$. Then $A_{w}$ is generated by $y_{1}^{ \pm 1}, \ldots, y_{m}^{ \pm 1}$ with relations $y_{i} y_{j}=q y_{j} y_{i}$ for all $i<j$. Set $S=\left\{s \in \mathbb{Z}^{m} \mid y^{s} \in Z\left(A_{w}\right)\right\}$, and observe that

$$
S=\left\{s \in \mathbb{Z}^{m} \mid \sum_{i<k} s_{i}-\sum_{i>k} s_{i}=0 \text { for all } k=1, \ldots, m\right\} .
$$

Since $m$ is odd, $S$ is generated by $(1,-1,1,-1, \ldots,-1,1)$. As in the proof of Theorem 4.4, it follows that $Z\left(A_{w}\right)$ is generated by $\left(y_{1} y_{2}^{-1} y_{3} y_{4}^{-1} \cdots y_{m-1}^{-1} y_{m}\right)^{ \pm 1}$. Therefore, in view of Proposition 1.3 and Theorem 4.4, $\operatorname{spec}_{w} A=\left\{J_{w}\right\} \cup \operatorname{prim}_{w} A$ and

$$
\operatorname{prim}_{w} A=\left\{J_{w}+\left\langle x_{l_{1}} x_{l_{3}} \cdots x_{l_{m}}-\lambda x_{l_{2}} x_{l_{4}} \cdots x_{l_{m-1}}\right\rangle \mid \lambda \in \mathbb{K}^{\times}\right\} .
$$

The patterns of links and cliques in spec $A$ are clear from Theorems 4.7 and 4.8 . When $w$ is nonempty and $n-\operatorname{card} w$ is odd, the cliques in $\operatorname{prim}_{w} A$ are infinite sequences with links in one or both directions. For instance, if $n=3$ the cliques of the maximal ideals $\left\langle x_{1}, x_{2}, x_{3}-1\right\rangle,\left\langle x_{1}, x_{2}-1, x_{3}\right\rangle$, and $\left\langle x_{1}-1, x_{2}, x_{3}\right\rangle$ have the following forms (with self-links deleted):

$$
\begin{aligned}
& \cdots \longrightarrow\left\langle x_{1}, x_{2}, x_{3}-q^{-1}\right\rangle \longrightarrow\left\langle x_{1}, x_{2}, x_{3}-1\right\rangle \longrightarrow\left\langle x_{1}, x_{2}, x_{3}-q\right\rangle \longrightarrow \cdots \\
& \cdots \rightleftarrows\left\langle x_{1}, x_{2}-q^{-1}, x_{3}\right\rangle \rightleftarrows\left\langle x_{1}, x_{2}-1, x_{3}\right\rangle \rightleftarrows\left\langle x_{1}, x_{2}-q, x_{3}\right\rangle \rightleftarrows \cdots \\
& \cdots \longleftarrow\left\langle x_{1}-q^{-1}, x_{2}, x_{3}\right\rangle \longleftarrow\left\langle x_{1}-1, x_{2}, x_{3}\right\rangle \longleftarrow\left\langle x_{1}-q, x_{2}, x_{3}\right\rangle \longleftarrow \cdots
\end{aligned}
$$




\section{Quantum Coordinate Rings of Semisimple Algebraic Groups}

We show that the quantum coordinate rings of semisimple algebraic groups fit the setup in (1.1). The required properties will be derived in the case that the parameter $q$ is an indeterminate, based on the development in Joseph's book [23]. For the case where $q$ is a scalar non-root of unity, see $[\mathbf{1 5}, \mathbf{1 6}, \mathbf{1 7}]$ and $[\mathbf{2 1}, \mathbf{2 2}]$. Throughout this section $\mathbf{g}, U_{q}(\mathbf{g}), G, R_{q}[G]$, and $W$ will be as in $(0.2)$ and $(0.3)$. In addition, $\pi=\left\{\alpha_{1}, \ldots, \alpha_{\ell}\right\}$ will be a set of simple roots for $\mathbf{g}, Q(\pi)=\mathbb{Z} \pi$ the corresponding root lattice, and $P(\pi)=\sum_{i=1}^{\ell} \mathbb{Z} \omega_{i}$ the corresponding weight lattice generated by the fundamental weights $\omega_{1}, \ldots, \omega_{\ell}$. The semigroup $\sum_{i=1}^{\ell} \mathbb{Z}_{\geq 0} \omega_{i}$ of dominant weights is denoted $P^{+}(\pi)$. The theory of highest weight modules for $U_{q}(\mathbf{g})$ closely resembles that for $\mathbf{g}$ (see [23, Section 4.3]). In particular, for each $\lambda \in P^{+}(\pi)$ there is a finite dimensional simple $U_{q}(\mathbf{g})$-module $V(\lambda)$ with highest weight $\lambda$, so that $V(\lambda)=U_{q}(\mathbf{g}) u_{\lambda}$ with $t_{i} \cdot u_{\lambda}=q^{\left(\alpha_{i}, \lambda\right)} u_{\lambda}$ for $i=1, \ldots, \ell$. Here $(-,-)$ denotes the Killing form for $\mathbf{g}$ and $t_{i} \in U^{0} \subseteq U_{q}(\mathbf{g})$ is the grouplike generator corresponding to $\alpha_{i}$. The complete set of isomorphism classes of finite dimensional simple $U_{q}(\mathbf{g})$-modules is $\left\{\gamma \otimes V(\lambda) \mid \gamma \in \Gamma ; \lambda \in P^{+}(\pi)\right\}$, where $\Gamma$ is the group of 1-dimensional $U_{q}(\mathbf{g})$-modules; in particular, $\Gamma \cong \mathbb{Z}_{2}^{\ell}$.

5.1. Let $\mathbb{K}$ be an extension field of a rational function field $k(q)$, where $k$ is a field of characteristic zero and $q$ is an indeterminate. We further assume that $\mathbb{K}$ contains $q^{1 / d}$, where $d$ is the determinant of the Cartan matrix of $\mathbf{g}$. Since the weight lattice $P(\pi)$ is contained in $\frac{1}{d} Q(\pi)$, inner products of weights lie in $\frac{1}{d} \mathbb{Z}$. Hence, by placing $q^{1 / d}$ in $\mathbb{K}$ we ensure that $q^{(\eta, \lambda)} \in \mathbb{K}$ for all $\eta, \lambda \in P(\pi)$.

Define the quantized enveloping algebra $U_{q}(\mathbf{g})[\mathbf{2 3}, 3.2 .9]$ over $\mathbb{K}$ rather than over $k(q)$, and let $A=R_{q}[G]$ be the corresponding $\mathbb{K}$-subalgebra of the Hopf dual $U_{q}(\mathbf{g})^{\star}[\mathbf{2 3}, 9.1 .1]$. (We will need to take $\mathbb{K}$ to be algebraically closed in order to obtain Assumption 7.) Thus $A$ is spanned by the coordinate functions $c_{\xi, v}^{V(\lambda)}$ for $\lambda \in P^{+}(\pi), \xi \in V(\lambda)^{*}, v \in V(\lambda)$; recall that $c_{\xi, v}^{V(\lambda)}$ is the element of $U_{q}(\mathbf{g})^{\star}$ given by the rule $c_{\xi, v}^{V(\lambda)}(u)=\xi(u v)$. The algebra $A$ is noetherian and satisfies the Nullstellensatz [23, 9.2.2]. In particular, Assumption 1 holds.

5.2. As noted in $[\mathbf{2 3}, 9.1 .1], A$ is a sub-Hopf algebra of $U_{q}(\mathbf{g})^{\star}$. Hence, the set $A^{\wedge}$ of linear characters on $A$ (i.e., $\mathbb{K}$-algebra homomorphisms $A \rightarrow \mathbb{K}$ ) forms a group under convolution (cf. $[\mathbf{2 3}, 1.3 .4])$. Further, there is a homomorphism $\theta^{\ell}: A^{\wedge} \rightarrow$ Aut $A$ and an anti-homomorphism $\theta^{r}: A^{\wedge} \rightarrow$ Aut $A$, where $\theta_{\chi}^{\ell}=\mu \circ(1 \otimes \chi) \circ \Delta$ and $\theta_{\chi}^{r}=\mu \circ(\chi \otimes 1) \circ \Delta$ for $\chi \in A^{\wedge}($ cf. $[23,1.3 .4])$; thus

$$
\theta_{\chi}^{\ell}(a)=\sum_{(a)} a_{1} \chi\left(a_{2}\right) \quad \text { and } \quad \theta_{\chi}^{r}(a)=\sum_{(a)} \chi\left(a_{1}\right) a_{2}
$$

for $a \in A$ and $\chi \in A^{\wedge}$. Observe that each $\theta_{\chi}^{\ell}$ commutes with each $\theta_{\chi}^{r}$.

For our particular algebra $A$, the group $A^{\wedge}$ is in fact a torus $[23,10.3 .8,10.3 .12]$; in particular, $A^{\wedge}$ is abelian. Hence, the sets $\mathcal{H}^{\ell}:=\left\{\theta_{\chi}^{\ell} \mid \chi \in A^{\wedge}\right\}$ and $\mathcal{H}^{r}:=\left\{\theta_{\chi}^{r} \mid\right.$ $\left.\chi \in A^{\wedge}\right\}$ are abelian subgroups of Aut $A$. Since these subgroups centralize each other, the set $\mathcal{H}:=\mathcal{H}^{\ell} \mathcal{H}^{r}$, finally, is also an abelian subgroup of Aut $A$. (Most of our assumptions hold equally well for $\mathcal{H}^{\ell}$ as for $\mathcal{H}$, but we will need $\mathcal{H}$ in order to obtain Assumption 3.) 
5.3. Let $\mathcal{W}=W \times W$, where $W$ denotes the Weyl group of $\mathbf{g}[\mathbf{2 3}, 4.2 .5]$, and write elements of $\mathcal{W}$ in the form $w=\left(w_{+}, w_{-}\right)$. By [23, 9.3.9], there is a disjoint partition $\operatorname{spec} A=\bigcup_{w \in \mathcal{W}} \mathbf{X}(w)$; thus set $\operatorname{spec}_{w} A=\mathbf{X}(w)$ for $w \in \mathcal{W}$. There is a unique minimum element $Q_{w} \in \mathbf{X}(w)[\mathbf{2 3}, 10.3 .5]$; hence, we obtain Assumption 2 with $J_{w}=Q_{w}$. (Cf. $[\mathbf{2 3}, 10.1 .8,10.1 .10]$ for a description of this ideal.)

In certain later formulas, the longest element of $W$ appears; let us denote this element $w_{0}$.

5.4. Each of the ideals $J_{w}$ is generated by certain elements of the form $c_{\eta, \lambda}^{\mu}[\mathbf{2 3}$, 10.1.10, 9.3.7, 9.1.5], all of which are $\mathcal{H}$-eigenvectors $[\mathbf{2 3}, 10.3 .12]$. Thus $J_{w}$ is $\mathcal{H}$-invariant. To see that $J_{w}$ is $\mathcal{H}$-polynormal, we first identify the appropriate automorphisms, as follows.

The presentation of $U_{q}(\mathbf{g})$ given in $[\mathbf{2 3}, 3.2 .9]$ includes commuting generators $t_{\alpha}^{ \pm 1}$ for $\alpha \in \pi$, such that $\Delta\left(t_{\alpha}^{ \pm 1}\right)=t_{\alpha}^{ \pm 1} \otimes t_{\alpha}^{ \pm 1}$. The map $\alpha \mapsto t_{\alpha}$ thus extends to a group homomorphism $\beta \mapsto t_{\beta}$ from the root lattice $Q(\pi)$ to the group of units of $U_{q}(\mathrm{~g})$, and $\Delta\left(t_{\beta}\right)=t_{\beta} \otimes t_{\beta}$ for all $\beta \in Q(\pi)$. Evaluation at $t_{\beta}$ gives a linear functional $\chi_{\beta} \in A^{*}$, and $\chi_{\beta}$ is a character because $\Delta\left(t_{\beta}\right)=t_{\beta} \otimes t_{\beta}$. To compute the effect of $\chi_{\beta}$, recall that $c_{\eta, \lambda}^{\mu}$ stands for an arbitrary element of the form $c_{\xi, v}^{V(\mu)}$ where $\mu \in P^{+}(\pi), \xi \in V(\mu)_{\eta}^{*}$ and $v \in V(\mu)_{\lambda}$. Since $v$ has weight $\lambda$, we have $t_{\beta} v=q^{(\lambda, \beta)} v$ and thus

$$
\chi_{\beta}\left(c_{\xi, v}^{V(\mu)}\right)=c_{\xi, v}^{V(\mu)}\left(t_{\beta}\right)=\xi\left(t_{\beta} v\right)=q^{(\lambda, \beta)} \xi(v) .
$$

Define automorphisms $\sigma_{\beta}^{\ell}=\theta_{\chi_{\beta}}^{\ell}$ and $\sigma_{\beta}^{r}=\theta_{\chi_{\beta}}^{r}$ in $\mathcal{H}$ for all $\beta \in Q(\pi)$. Using the comultiplication formulas for the $c_{\eta, \lambda}^{\mu}[\mathbf{2 3}, 1.4 .7]$, one obtains $\sigma_{\beta}^{\ell}\left(c_{\xi, v}^{V(\mu)}\right)=q^{(\lambda, \beta)} c_{\xi, v}^{V(\mu)}$ and $\sigma_{\beta}^{r}\left(c_{\xi, v}^{V(\mu)}\right)=q^{(\eta, \beta)} c_{\xi, v}^{V(\mu)}$.

We also require analogous automorphisms arising from weights. As in [25, 3.1], $U_{q}(\mathbf{g})$ can be enlarged to a Hopf algebra $\check{U}=U^{-} \check{U}^{0} U^{+}$where $\check{U}^{0}$ is the group algebra of the multiplicative group $\tau(P(\pi))$ isomorphic to $P(\pi)$; here $\tau(\alpha)=t_{\alpha}$ for $\alpha \in Q(\pi)$ and the new $\tau(\lambda)$ 's satisfy analogous relations:

$$
\begin{aligned}
\tau(\lambda) x_{\alpha} \tau(\lambda)^{-1} & =q^{(\alpha, \lambda)} x_{\alpha}, \\
\tau(\lambda) y_{\alpha} \tau(\lambda)^{-1} & =q^{-(\alpha, \lambda)} y_{\alpha}, \\
\Delta \tau(\lambda) & =\tau(\lambda) \otimes \tau(\lambda)
\end{aligned}
$$

for $\lambda \in P(\pi)$ and $\alpha \in Q(\pi)$. (This is a smaller version of the algebra labelled $\check{U}$ in $[\mathbf{2 3}, 3.2 .10]$.) The $U_{q}(\mathbf{g})$-modules $V(\mu)$ all become $\check{U}$-modules with the same weight spaces as originally, and $A$ becomes a subalgebra of $\check{U}^{\star}$. Consequently, for any $\beta \in P(\pi)$ we obtain a character $\chi_{\beta}$ of $A$ as above, and corresponding automorphisms $\sigma_{\beta}^{\ell}$ and $\sigma_{\beta}^{r}$. Since the formulas for $\sigma_{\beta}^{\ell}$ and $\sigma_{\beta}^{r}$ applied to elements of the form $c_{\xi, v}^{V(\mu)}$ only depend on the weights of $\xi$ and $v$, these automorphisms are completely determined by the following expressions:

$$
\sigma_{\beta}^{\ell}\left(c_{\eta, \lambda}^{\mu}\right)=q^{(\lambda, \beta)} c_{\eta, \lambda}^{\mu} \quad \text { and } \quad \sigma_{\beta}^{r}\left(c_{\eta, \lambda}^{\mu}\right)=q^{(\eta, \beta)} c_{\eta, \lambda}^{\mu}
$$

for $\mu \in P^{+}(\pi)$ and $\eta, \lambda \in \Omega(V(\mu))$.

By $[\mathbf{2 3}, 10.1 .10,10.1 .8,9.1 .6]$, the ideal $J_{w}$ for $w \in \mathcal{W}$ can be described as follows:

$$
J_{w}=Q_{w}=\sum_{\lambda \in P^{+}(\pi)} A V_{w_{+}}^{+}(\lambda)^{\perp}+\sum_{\lambda \in P^{+}(\pi)} A V_{w_{-}}^{-}(\lambda)^{\perp}
$$


where

$$
\begin{aligned}
V_{w_{+}}^{+}(\lambda)^{\perp} & =\left\{c_{\xi, u_{\lambda}}^{V(\lambda)} \mid \xi \in\left(U_{q}\left(\mathbf{b}^{+}\right) u_{w_{+} \lambda}\right)^{\perp} \subseteq V(\lambda)^{*}\right\}, \\
V_{w_{-}}^{-}(\lambda)^{\perp} & =\left\{c_{\xi, u_{w_{0} \lambda}}^{V(\lambda)} \mid \xi \in\left(U_{q}\left(\mathbf{b}^{-}\right) u_{w_{-} w_{0} \lambda}\right)^{\perp} \subseteq V(\lambda)^{*}\right\},
\end{aligned}
$$

and $u_{\lambda}, u_{w_{0} \lambda}, u_{w_{+} \lambda}, u_{w_{-} w_{0} \lambda}$ are nonzero vectors in $V(\lambda)$ of weights $\lambda, w_{0} \lambda, w_{+} \lambda$, $w_{-} w_{0} \lambda$ respectively. To prove that $J_{w}$ is polynormal requires suitable commutation formulas for the elements $c_{\xi, u_{\lambda}}^{V(\lambda)}$ and $c_{\xi, u_{w_{0} \lambda}}^{V(\lambda)}$. The formulas given in [23, 9.1.5] are of the right type but not sharp enough for the purpose; instead, we use $[\mathbf{1 7}, 3.10]$, observing that the proof given there, over $\mathbb{C}$, works equally well over $\mathbb{K}$. Translating this result into the notation of $[\mathbf{2 3}]$ yields that

$$
c_{\eta^{\prime}, \lambda^{\prime}}^{\mu} c_{\xi, u_{\lambda}}^{V(\lambda)} \equiv q^{\left(\lambda^{\prime}, \lambda\right)-\left(\eta^{\prime}, \eta\right)} c_{\xi, u_{\lambda}}^{V(\lambda)} c_{\eta^{\prime}, \lambda^{\prime}}^{\mu} \quad \bmod \sum_{\nu \in Q^{+}(\pi) \backslash\{0\}}\left\langle c_{\rho, u_{\lambda}}^{V(\lambda)} \mid \rho \in\left(\xi \cdot U_{q}\left(\mathbf{b}^{+}\right)\right)_{\eta-\nu}\right\rangle
$$

for $\xi \in V(\lambda)_{\eta}^{*}, \mu \in P^{+}(\pi)$ and $\eta^{\prime}, \lambda^{\prime} \in \Omega(V(\mu))$. Hence,

$$
a c_{\xi, u_{\lambda}}^{V(\lambda)} \equiv c_{\xi, u_{\lambda}}^{V(\lambda)} \sigma_{\lambda}^{\ell} \sigma_{-\eta}^{r}(a) \quad \bmod \sum_{\nu \in Q^{+}(\pi) \backslash\{0\}}\left\langle c_{\rho, u_{\lambda}}^{V(\lambda)} \mid \rho \in\left(\xi . U_{q}\left(\mathbf{b}^{+}\right)\right)_{\eta-\nu}\right\rangle
$$

for $a \in A$. It follows that $A V_{w_{+}}^{+}(\lambda)^{\perp}$ is an $\mathcal{H}$-polynormal ideal of $A$.

Similarly, $A V_{w_{-}}^{-}(\lambda)^{\perp}$ is an $\mathcal{H}$-polynormal ideal. This follows from the commutation formula

$$
\begin{aligned}
c_{\xi, u_{w_{0} \lambda}}^{V(\lambda)} c_{\eta^{\prime}, \lambda^{\prime}}^{\mu} \equiv q^{\left(\lambda^{\prime}, w_{0} \lambda\right)-\left(\eta^{\prime}, \eta\right)} c_{\eta^{\prime}, \lambda^{\prime}}^{\mu} c_{\xi, u_{w_{0} \lambda}}^{V(\lambda)} & \bmod \sum_{\nu \in Q^{+}(\pi) \backslash\{0\}}\left\langle c_{\rho, u_{w_{0} \lambda}}^{V(\lambda)} \mid \rho \in\left(\xi . U_{q}\left(\mathbf{b}^{-}\right)\right)_{\eta+\nu}\right\rangle
\end{aligned}
$$

for $\xi \in V(\lambda)_{\eta}^{*}, \mu \in P^{+}(\pi)$ and $\eta^{\prime}, \lambda^{\prime} \in \Omega(V(\mu))$, which can be obtained exactly as $[\mathbf{1 7}, 3.10]$, and which implies

$$
c_{\xi, u_{w_{0} \lambda}}^{V(\lambda)} a \equiv \sigma_{w_{0} \lambda}^{\ell} \sigma_{-\eta}^{r}(a) c_{\xi, u_{w_{0} \lambda}}^{V(\lambda)} \quad \bmod \sum_{\nu \in Q^{+}(\pi) \backslash\{0\}}\left\langle c_{\rho, u_{w_{0} \lambda}}^{V(\lambda)} \mid \rho \in\left(\xi \cdot U_{q}\left(\mathbf{b}^{-}\right)\right)_{\eta+\nu}\right\rangle
$$

for $a \in A$.

Therefore $J_{w}$ is $\mathcal{H}$-polynormal, and Assumption 3 is established.

5.5. It is convenient to note that the set

$$
\mathcal{H}_{\pi}:=\left\{\sigma_{\lambda}^{\ell} \sigma_{\eta}^{r} \mid \lambda, \eta \in P(\pi) \text { and } \lambda+\eta \in Q(\pi)\right\}
$$

is a subgroup of $\mathcal{H}$, and that the ideals $J_{w}$ are $\mathcal{H}_{\pi}$-polynormal. (The latter assertion follows from the fact that the automorphisms implementing $\mathcal{H}$-polynormality in (5.4) are all of the form $\left(\sigma_{\lambda}^{\ell} \sigma_{-\eta}^{r}\right)^{-1}$ or $\sigma_{w_{0} \lambda}^{\ell} \sigma_{-\eta}^{r}$ for $\lambda \in P^{+}(\pi)$ and $\eta \in \Omega(V(\lambda))$.) Since the inner product of a weight with a root is integral, we have

$$
\sigma_{\lambda}^{\ell} \sigma_{\eta}^{r}\left(c_{\eta^{\prime}, \lambda^{\prime}}^{\mu}\right)=q^{\left(\lambda^{\prime}-\eta^{\prime}, \lambda\right)+\left(\eta^{\prime}, \lambda+\eta\right)} c_{\eta^{\prime}, \lambda^{\prime}}^{\mu} \in q^{\mathbb{Z}} c_{\eta^{\prime}, \lambda^{\prime}}^{\mu}
$$

for all $\sigma_{\lambda}^{\ell} \sigma_{\eta}^{r} \in \mathcal{H}_{\pi}, \mu \in P^{+}(\pi)$, and $\eta^{\prime}, \lambda^{\prime} \in \Omega(V(\mu))$. Thus $A$ is spanned by $\mathcal{H}_{\pi^{-}}$ eigenvectors, and the eigenvalues of any $\sigma \in \mathcal{H}_{\pi}$ are integral powers of $q$. Since $q$ is not a root of unity, it thus follows from Lemma 4.6 that the $\langle\sigma\rangle$-orbit of any ideal in $A$ is either infinite or a singleton. 
5.6. For $w \in \mathcal{W}$, there is an Ore set $b_{w}$ in $A / J_{w}=R_{q}[G] / Q_{w}$ which determines $\operatorname{spec}_{w} A=\mathbf{X}(w)$. More precisely, $\operatorname{spec}_{w} A \cong \operatorname{spec}\left(A / J_{w}\right)\left[b_{w}^{-1}\right]$ via the localization map $[\mathbf{2 3}, 10.3 .2]$; that is,

$$
\left\{P / J_{w} \mid P \in \operatorname{spec}_{w} A\right\}=\left\{Q \in \operatorname{spec} A / J_{w} \mid Q \cap b_{w}=\varnothing\right\} .
$$

The elements of $b_{w}$ are nonzero scalar multiples of the images in $A / J_{w}$ of the elements

$$
b_{w, \lambda}=c_{w_{+} \lambda} d_{w_{-} \lambda_{0}}=c_{w_{+} \lambda, \lambda}^{\lambda} c_{w_{-} w_{0} \lambda_{0}, w_{0} \lambda_{0}}^{\lambda_{0}}
$$

for $\lambda \in P^{+}(\pi)$, where $\lambda_{0}=-w_{0} \lambda$. By [23, 9.1.5], the two factors of $b_{w, \lambda}$ are normal modulo $J_{\lambda}^{+}\left(w_{+} \lambda, \lambda\right)$ and $J_{\lambda_{0}}^{-}\left(w_{-} w_{0} \lambda_{0}, w_{0} \lambda_{0}\right)$ respectively. Since both of these ideals are contained in $J_{w}[\mathbf{2 3}, 10.1 .10]$, we have that $b_{w, \lambda}$ is normal modulo $J_{w}$.

Let $E_{w}$ denote the multiplicative set in $A / J_{w}$ generated by the images of the elements $b_{w, \alpha}$ for $\alpha \in \pi$; thus $E_{w}$ is an Ore set generated by a finite set of normal $\mathcal{H}$-eigenvectors. Modulo $J_{w}$, the elements $c_{w_{+} \lambda}$ and $d_{w_{-} \mu}$ commute up to scalars $[\mathbf{2 3}, 9.1 .5]$, and hence it follows from $[\mathbf{2 3}, 9.1 .10]$ that $b_{w, \lambda+\mu}$ is congruent modulo $J_{w}$ to a nonzero scalar multiple of $b_{w, \lambda} b_{w, \mu}$ for all $\lambda, \mu \in P^{+}(\pi)$. Hence,

$$
b_{w}=\left\{k e \mid k \in \mathbb{K}^{\times} \text {and } e \in E_{w}\right\},
$$

and Assumptions 4,5 follow. Also, $A_{w}=\left(A / J_{w}\right)\left[E_{w}^{-1}\right]$ coincides with $\left(A / J_{w}\right)\left[b_{w}^{-1}\right]$, which is denoted $R_{w}$ in $[\mathbf{2 3}, 10.3 .2]$; for the convenience of the reader we use the latter notation.

5.7. By $[\mathbf{2 3}, 9.3 .16]$, there is a grading of $A$ by the finite group $\Gamma=P(\pi) / 2 P(\pi)$ satisfying the conditions of $[\mathbf{2 3}, 1.3 .8]$. In particular, there is a homomorphism $\Gamma \stackrel{\cong}{\rightrightarrows} \Gamma^{\wedge} \rightarrow A^{\wedge}$ which allows $\Gamma$ to act on (ideals of) $A$ via automorphisms of the form $\theta_{\chi_{\gamma}}^{\ell}$ in $\mathcal{H}^{\ell}$. Since each $\operatorname{spec}_{w} A$ is stable under $\mathcal{H}$ (by Assumptions 4,5), it follows that $\operatorname{spec}_{w} A$ is stable under the action of $\Gamma$.

Let $R_{0}$ be the zero component for the $\Gamma$-grading of $A$, fix $w \in \mathcal{W}$, and let $B_{w}$ be the subalgebra of $R_{w}$ defined in $[\mathbf{2 3}, 10.3 .3]$. Then $\left(\left(R_{0}+Q_{w}\right) / Q_{w}\right)\left[b_{w}^{-1}\right]=B_{w}\left[b_{w}\right]$ by $[\mathbf{2 3}, 10.3 .4]$. Now take $P \in \mathbf{X}(w)$. Then $P \cap R_{0}$ is an $(\operatorname{ad} R)$-stable prime ideal of $R_{0}\left[\mathbf{2 3}, 10.3 .4\right.$, end], and so $\left(P \cap R_{0}\right) A$ is a semiprime ideal of $A$ and the primes of $A$ minimal over $\left(P \cap R_{0}\right) A$ form a single $\Gamma$-orbit $[\mathbf{2 3}, 1.3 .9]$. Since $A$ is a finite module over $R_{0}$, one of the primes minimal over $\left(P \cap R_{0}\right) A$ is $P$ itself (cf. $[\mathbf{1 3}, 10.6])$. Hence, all the primes minimal over $\left(P \cap R_{0}\right) A$ lie in $\mathbf{X}(w)$. It follows that $A /\left(P \cap R_{0}\right) A$ is $b_{w}$-torsionfree, and consequently $\left(P \cap R_{0}\right) R_{w}$ is a semiprime ideal of $R_{w}[\mathbf{1 3}, 9.20]$. Note that $P R_{w}$ is minimal over $\left(P \cap R_{0}\right) R_{w}$, and that $\left(P \cap R_{0}\right) R_{w}=\left(P R_{w} \cap B_{w}\right) R_{w}$. Further, $P R_{w} \cap B_{w}$ is generated by its intersection with the algebra $Z_{w} \subseteq Z\left(R_{w}\right)[\mathbf{2 3}, 10.3 .3]$. This shows that $P R_{w}$ is minimal over a centrally generated semiprime ideal of $R_{w}$, and thus verifies Assumption 6. In the case $\mathbb{K}=\mathbb{C}$, one in fact has that all ideals of $A_{w}$ are centrally generated $[\mathbf{1 7}, 4.15]$.

Finally, $[\mathbf{2 3}, 10.3 .8]$ shows that if $\mathbb{K}$ is algebraically closed, then $\mathcal{H}$ (in fact, $\mathcal{H}^{\ell}$ ) acts transitively on $\max R_{w}$. In view of Corollary 1.4, Assumption 7 follows in this case.

5.8. All of Assumptions $1-7$ are now verified for the algebra $A=R_{q}[G]$. Immediate consequences include the following applications of Proposition 1.7 and Theorems $2.6,3.4,3.5$. (In obtaining part (b) from Theorem 2.6, we use the fact that $R_{q}[G]$ is a domain $[\mathbf{2 3}, 9.1 .9]$.) 
Theorem. (a) $\operatorname{spec} R_{q}[G]$ has normal separation, and therefore $R_{q}[G]$ satisfies the strong second layer condition.

(b) Every nonzero prime ideal in $R_{q}[G]$ is linked to itself.

(c) If $\mathbb{K}$ is algebraically closed, then each clique in $\operatorname{spec} R_{q}[G]$ is either infinite or a singleton.

(d) If $\mathbb{K}$ is algebraically closed, then the intersection of any clique in $\operatorname{spec} R_{q}[G]$ is a localizable prime ideal.

We note that the second layer condition for the cliques of the augmentation ideals in the algebras $R_{q}\left[S L_{n}(\mathbb{K})\right], R_{q}\left[G L_{n}(\mathbb{K})\right]$, and $\mathcal{O}_{q}\left(M_{n}(\mathbb{K})\right)$ was verified by Chin and Musson [5, Corollary 1.4].

5.9. For the remainder of the section, we assume that $\mathbb{K}$ is algebraically closed.

Let $w \in \mathcal{W}$. Recall from (5.4) that $J_{w}$ is generated by the set

$$
\begin{aligned}
& \left\{c_{\xi, u_{\lambda}}^{V(\lambda)} \mid \lambda \in P^{+}(\pi) ; \xi \in\left(U_{q}\left(\mathbf{b}^{+}\right) u_{w_{+} \lambda}\right)^{\perp}\right\} \\
& \quad \cup\left\{c_{\xi, u_{w_{0} \lambda}}^{V(\lambda)} \mid \lambda \in P^{+}(\pi) ; \xi \in\left(U_{q}\left(\mathbf{b}^{-}\right) u_{w_{-} w_{0} \lambda}\right)^{\perp}\right\} .
\end{aligned}
$$

In fact, it suffices to let $\lambda$ run through the fundamental weights $\omega_{1}, \ldots, \omega_{\ell}[\mathbf{2 4}$, Théorème 3]. A suitable finite set of these generators, taken in an appropriate order, forms an $\mathcal{H}$-polynormal sequence of generators $x_{w, 1}, \ldots, x_{w, m(w)}$ for $J_{w}$. Each $x_{w, j}$ is $h_{w, j}$-normal modulo $\left\langle x_{w, 1}, \ldots, x_{w, j-1}\right\rangle$ for some $h_{w, j} \in \mathcal{H}$, and in view of (5.4) we can take these $h_{w, j}$ from the (finite) set

$$
\left\{\sigma_{-\omega_{i}}^{\ell} \sigma_{\eta}^{r}, \sigma_{w_{0} \omega_{i}}^{\ell} \sigma_{-\eta}^{r} \mid i=1, \ldots, \ell ; \eta \in \Omega\left(V\left(\omega_{i}\right)\right)\right\} \subseteq \mathcal{H}_{\pi} .
$$

Theorem 2.11 shows that all non-self links in $\operatorname{spec}_{w} A$ can be expressed in terms of the automorphisms $h_{w, j}$ : if $P$ and $Q$ are distinct primes in $\operatorname{spec}_{w} A$ such that $P \rightsquigarrow Q$, then $P=h_{w, j}(Q)$ for some $j$.

For the maximal ideals of codimension 1 , an alternate description of the links was given by Musson in [32, 2.2].

5.10. By Theorem 3.3, the subgroup $T_{w} \subseteq \mathcal{H}$ determines all the cliques within $\operatorname{spec}_{w} A$, the set $\Sigma_{w} \cap\left\{h_{w, 1}, \ldots, h_{w, m(w)}\right\}$ contains a full set of coset representatives for $\Sigma_{w} / N_{w}$, and this set generates $T_{w}$ modulo $N_{w}$. By (5.5) and Theorem $3.4, T_{w} / N_{w}$ is free abelian of finite rank. There are natural bijections $T_{w} / N_{w} \leftrightarrow$ clique $(P)$ for all $P \in \operatorname{prim}_{w} A$ (Proposition 3.1(b)), and natural surjections $T_{w} / N_{w}$ $\rightarrow$ clique $(P)$ for all $P \in \operatorname{spec}_{w} A$ (Theorem 3.3(a)). Thus any bound on the rank of $T_{w} / N_{w}$ gives a bound on the sizes of these cliques.

5.11. To develop a bound on the rank of $T_{w} / N_{w}$, we work with the algebras $R_{0}$, $B_{w}, Z_{w}$ discussed in (5.7). Recall that $Z_{w} \subseteq Z\left(R_{w}\right)$ and that $Z_{w}$ is generated by certain elements of the form

$$
a_{w, \mu}=c_{w_{+} \mu}^{-1} d_{w_{-} \mu_{0}}=\left(c_{w_{+} \mu, \mu}^{\mu}\right)^{-1} c_{w_{-} w_{0} \mu_{0}, w_{0} \mu_{0}}^{\mu_{0}},
$$

where $\mu \in P^{+}(\pi)$ and $\mu_{0}=-w_{0} \mu[\mathbf{2 3}, 10.3 .3]$. Since the $a_{w, \mu}$ are $\mathcal{H}$-eigenvectors, $Z_{w}$ is invariant under $\mathcal{H}$. 
Lemma. The homomorphism $T_{w} \rightarrow$ Aut $Z_{w}$ afforded by restriction (of the action of $\mathcal{H}$ on $R_{w}$ ) has kernel precisely $N_{w}$.

Proof. By Theorem 3.3(a), all primes in $\operatorname{spec} R_{w}$ are invariant under $N_{w}$. Since every prime of $Z_{w}$ is the contraction of some prime of $R_{w}[\mathbf{2 3}, 10.3 .4]$, it follows that all primes of $Z_{w}$ are invariant under $N_{w}$. Now $Z_{w}$ is a Laurent polynomial ring over $\mathbb{K}$ by $[\mathbf{2 3}, 10.3 .3]$. Hence, every maximal ideal $M$ of $Z_{w}$ has codimension 1 , that is, $Z_{w}=M+\mathbb{C} \cdot 1$. Consequently, $\eta(t)-t \in M$ for all $\eta \in N_{w}$ and $t \in Z_{w}$. Since $J\left(Z_{w}\right)=0$, it follows that $N_{w}$ acts trivially on $Z_{w}$.

Conversely, let $\eta \in T_{w}$ be an automorphism that acts trivially on $Z_{w}$. Then by $[\mathbf{2 3}, 10.3 .4], \eta$ permutes each $\Gamma$-orbit in $\mathbf{X}(w)$. Therefore $\eta^{|\Gamma| !}$ fixes each prime in $\mathbf{X}(w)$, and so $\eta^{|\Gamma| !} \in N_{w}$. Since $T_{w} / N_{w}$ is torsionfree, we conclude that $\eta \in N_{w}$, and the proof is complete.

5.12. Theorem. Let $w \in \mathcal{W}$. Then the free abelian group $T_{w} / N_{w}$ has rank at most $\ell-s(w)$, where $\ell=\operatorname{rank} G$ and $s(w)$ is the minimum length of an expression for $w_{-}^{-1} w_{+}$as a product of reflections. Consequently, for all $P \in \operatorname{spec}_{w} A$ the rank of $T_{P} / N_{P}$ is at most $\ell-s(w)$.

Proof. The groups $T_{w} / N_{w}$ and $T_{P} / N_{P}$ are free abelian of finite rank by (5.5) and Theorem 3.4, and $T_{P} / N_{P}$ is a homomorphic image of $T_{w} / N_{w}$ by Theorem 3.3(c). Hence, it suffices to show that $\operatorname{rank} T_{w} / N_{w} \leq \ell-s(w)$.

By $[\mathbf{2 3}, 10.3 .3], Z_{w}$ is a Laurent polynomial ring of the form $\mathbb{K}\left[a_{w, \mu_{1}}^{ \pm 1}, \ldots, a_{w, \mu_{r}}^{ \pm 1}\right]$ where $\mu_{1}, \ldots, \mu_{r}$ is a basis for $P_{w}(\pi)$. It is shown in [23, A.1.18] that $r=\operatorname{rank} P_{w}(\pi)$ $=\ell-s(w)$. There are commuting $\mathbb{K}$-algebra automorphisms $\eta_{1}, \ldots, \eta_{r}$ of $Z_{w}$ such that $\eta_{i}\left(a_{w, \mu_{j}}\right)=q^{\delta_{i j}} a_{w, \mu_{j}}$ for all $i, j$, and the $\eta_{i}$ generate a free abelian subgroup of Aut $Z_{w}$ of rank $r$.

We have just seen that restriction affords an embedding $T_{w} / N_{w} \rightarrow$ Aut $Z_{w}$. As noted in (5.9) and (5.10), $T_{w} / N_{w}$ can be generated by cosets of the form $h_{w, k} N_{w}$ for certain $h_{w, k} \in \mathcal{H}_{\pi}$. Observe that the elements $a_{w, \mu_{j}}$ are eigenvectors for the $h_{w, k}$, with all the eigenvalues being powers of $q$. Thus, restriction actually yields an embedding of $T_{w} / N_{w}$ into $\left\langle\eta_{1}, \ldots, \eta_{r}\right\rangle$, and therefore $\operatorname{rank} T_{w} / N_{w} \leq r$, as desired. $\square$

5.13. Example 6.12 shows that the bound $\ell-s(w)$ is best possible in general, for in this case $s(w)=0$ and $\operatorname{rank} T_{w} / N_{w}=\ell$. On the other hand, the case $w=((1,2),(1,2))$ of Example 6.8 demonstrates that in general the inequality $\operatorname{rank} T_{w} / N_{w} \leq \ell-s(w)$ can be strict. In that case, $\operatorname{prim}_{w} A$ consists of localizable primes, each having a singleton clique, whence $T_{w} / N_{w}$ is trivial, whereas $\ell-s(w)=1-0=1$.

It would be very interesting to find a general formula giving the rank of $T_{w} / N_{w}$ in terms of combinatorial properties of $w$.

\section{The Quantum Special Linear Groups}

In this final section, we specialize some of the results of the previous section to the groups $G=S L_{n}(\mathbb{C})$. That allows us to give these results in a more concrete form, using the standard presentation of $R_{q}\left[S L_{n}(\mathbb{C})\right]$ by generators and relations. This setting also allows us to do detailed calculations of some examples which illustrate and support our results.

Throughout this section, we let $A=R_{q}\left[S L_{n}(\mathbb{C})\right]$. Thus, we are in the setting of Section 5 with $\mathbb{K}=\mathbb{C}$ and $q \in \mathbb{C}$ transcendental over $\mathbb{Q}$. We shall cite various 
facts from $[\mathbf{1 5}, \mathbf{1 6}]$; in order to match notation with that of $[\mathbf{2 3}]$ on which Section 5 relies, we replace all occurrences of $q^{2}$ in $[\mathbf{1 5}, \mathbf{1 6}]$ by $q$. The results of Theorems 5.8 and 5.12 hold for $A$; in particular, spec $A$ has normal separation and $A$ satisfies the strong second layer condition. These results can also be extended to the algebras $R_{q}\left[G L_{n}(\mathbb{C})\right] ;$ see $(6.14)$.

6.1. It is well known (cf. $[\mathbf{1 5}, 1.4 .1]$ ) that $A$ is generated by symbols $X_{i j}$ for $1 \leq$ $i, j \leq n$ subject to the following relations:

$$
\begin{gathered}
x_{i j} x_{l j}=q x_{l j} x_{i j} \quad(i<l), \\
x_{i j} x_{i m}=q x_{i m} x_{i j} \quad(j<m), \\
x_{i m} x_{l j}=x_{l j} x_{i m} \quad(i<l \text { and } j<m), \\
x_{i j} x_{l m}-x_{l m} x_{i j}=\left(q-q^{-1}\right) x_{i m} x_{l j} \quad(i<l \text { and } j<m), \\
\operatorname{Det}_{q} X:=\sum_{\sigma \in S_{n}}(-q)^{l(\sigma)} x_{\sigma(1), 1} x_{\sigma(2), 2} \cdots x_{\sigma(n), n}=1 .
\end{gathered}
$$

Further, $A$ is a Hopf algebra with comultiplication $\Delta$ and counit $\epsilon$ given by

$$
\Delta\left(X_{i j}\right)=\sum_{l=1}^{n} X_{i l} \otimes X_{l j} \quad \text { and } \quad \epsilon\left(X_{i j}\right)=\delta_{i j}
$$

for all $i, j$. For a description of the antipode, which we shall not need here, see $[\mathbf{1 5}$, 1.4.1]. The algebra $\mathcal{O}_{q}\left(M_{n}(\mathbb{C})\right)$, the coordinate ring of quantum $n \times n$ matrices, is generated by symbols $X_{i j}$ satisfying the relations above except that $\operatorname{Det}_{q} X$ is not set equal to 1.

Recall from [28] that $A$ is a domain, and from [12,3.3] that - due to our assumption that $q$ is not a root of unity - all prime ideals in $A$ are completely prime.

6.2. The character group $A^{\wedge}$ is isomorphic, via evaluation at the matrix $\left(X_{i j}\right)$, to the torus of diagonal matrices in $S L_{n}(\mathbb{C})[\mathbf{1 5}, 2.5]$. For $\chi \in A^{\wedge}$, the automorphisms $\theta_{\chi}^{\ell}$ and $\theta_{\chi}^{r}$ act on $\left(X_{i j}\right)$ via multiplication by the diagonal matrix $\left(\chi\left(X_{i j}\right)\right)$ :

$$
\theta_{\chi}^{\ell}\left(X_{i j}\right)=X_{i j} \chi\left(X_{j j}\right) \quad \text { and } \quad \theta_{\chi}^{r}\left(X_{i j}\right)=\chi\left(X_{i i}\right) X_{i j}
$$

for all $i, j$.

6.3. (a) Certain of the coordinate elements $c_{\eta, \lambda}^{\mu}$ coincide with quantum minors, i.e., quantum determinants of submatrices of the matrix $\left(X_{i j}\right)$. These are the coordinate elements

$$
c_{k, w}^{+}:=c_{w \varpi_{k}, \varpi_{k}}^{\varpi_{k}} \quad \text { and } \quad c_{k, w}^{-}:=c_{w w_{0} \varpi_{n-k}, w_{0} \varpi_{n-k}}^{\varpi_{n-k}}
$$

for $k=1, \ldots, n-1$ and $w \in W$ (suitably normalized), where $\varpi_{1}, \ldots, \varpi_{n-1}$ are the fundamental dominant weights (cf. $[\mathbf{1 5}, 1.5]$ ). Recall that the Weyl group $W$ may be identified with the symmetric group $S_{n}[\mathbf{1 5}, 1.5]$. Hence, $\mathcal{W}=S_{n} \times S_{n}$.

(b) Let $K$ and $L$ be nonempty subsets of $\{1, \ldots, n\}$ of the same cardinality, say cardinality $t$. Set $D(K, L)$ equal to the quantum minor built from the $X_{i j}$ for $i \in K$ and $j \in L$, as in $[\mathbf{3 4}, 4.3]$ (cf. [33, 1.2]). 
List the elements of $K$ and $L$ in ascending order; say $K=\left\{k_{1}<k_{2}<\cdots<k_{t}\right\}$ and $L=\left\{l_{1}<l_{2}<\cdots<l_{t}\right\}$. Then define $K \leq L$ if and only if $\left(k_{1}, k_{2}, \ldots, k_{t}\right) \leq$ $\left(l_{1}, l_{2}, \ldots, l_{t}\right)$ with respect to the product ordering, i.e., if and only if $k_{s} \leq l_{s}$ for $s=1, \ldots, t$.

(c) Let $j \in\{1, \ldots, n-1\}$ and $w \in S_{n}$. Set $J=\{1, \ldots, j\}$ and $J^{\prime}=\{j+1, \ldots, n\}$. Then by $[\mathbf{1 5}, 1.5]$,

$$
c_{j, w}^{+}=D(w J, J) \quad \text { and } \quad c_{j, w}^{-}=D\left(w J^{\prime}, J^{\prime}\right) .
$$

For $y, z \in S_{n}$, define $y \leq_{j} z$ if and only if $y J \leq z J$. Note that $\leq_{j}$ is only a pre-order (reflexive and transitive, but not antisymmetric). These orderings can be interpreted as component suborderings of the reverse Bruhat ordering on the Weyl group $W[\mathbf{1 5}, 1.8 ; \mathbf{1 6}, \S 1]$ (cf. [18, Example 2, p. 119]).

(d) For $w \in W$ and $\lambda \in P^{+}(\pi)$, observe that the ideals $I^{ \pm}(w, \lambda)$ defined in $[\mathbf{1 5}$, 1.7] can be expressed in the forms

$$
I^{+}(w, \lambda)=\left\langle V_{w}^{+}(\lambda)^{\perp}\right\rangle \quad \text { and } \quad I^{-}(w \lambda)=\left\langle V_{w}^{-}\left(-w_{0} \lambda\right)^{\perp}\right\rangle
$$

(recall (5.4)). It is shown in $[\mathbf{1 5}, 2.2]$ that

$$
\left.I^{ \pm}\left(w, \varpi_{j}\right)=\left\langle c_{j, y}^{ \pm}\right| y \in S_{n} \text { and } y \not_{j} w\right\rangle
$$

for $j=1, \ldots, n-1$. Now set $I_{w}^{ \pm}=\sum_{j=1}^{n-1} I^{ \pm}\left(w, \varpi_{j}\right)$ and $\mathcal{E}_{w}^{ \pm}=\left\{c_{j, w}^{ \pm} \mid j=\right.$ $1, \ldots, n-1\}$. Given $w=\left(w_{+}, w_{-}\right) \in \mathcal{W}$, set $I_{w}=I_{w_{+}}^{+}+I_{w_{-}}^{-}$and $\mathcal{E}_{w}=\mathcal{E}_{w_{+}}^{+} \cup \mathcal{E}_{w_{-}}^{-}$. (The definition of $I_{w}$ given in [16, p. 458] relies on the alternate description of the $I^{ \pm}\left(w, \varpi_{j}\right)$ displayed above.) Since $-w_{0} \varpi_{j}=\varpi_{n-j}$ for all $j$, we have

$$
I_{w}=\sum_{j=1}^{n-1}\left(\left\langle V_{w_{+}}^{+}\left(\varpi_{j}\right)^{\perp}\right\rangle+\left\langle V_{w_{-}}^{-}\left(\varpi_{n-j}\right)^{\perp}\right\rangle\right) .
$$

It then follows from [24, Théorème 3] and the definition of $Q_{w}$ (cf. (5.4)) that $I_{w}=Q_{w}$, which yields the following useful description of $Q_{w}$ :

$$
Q_{w}=I_{w}=I_{w_{+}}^{+}+I_{w_{-}}^{-},
$$

where

$$
I_{w_{ \pm}}^{ \pm}=\left\langle c_{j, y}^{ \pm} \mid j=1, \ldots, n-1 ; y \in S_{n} ; y \not \Varangle_{j} w_{ \pm}\right\rangle .
$$

6.4. We next show that our notation $\operatorname{spec}_{w} A$ as used in Section 5 coincides with the use of this notation in $[\mathbf{1 5}, \mathbf{1 6}]$.

Proposition. The subsets $\mathbf{X}(w) \subseteq \operatorname{spec} A$ as defined in [23, 9.3.9] coincide with the sets $\mathrm{spec}_{w} A$ defined in $[\mathbf{1 5}, 2.8 ; \mathbf{1 6}, 1.3]$.

Proof. Take the form of the definition of $\operatorname{spec}_{w} A$ given in $[\mathbf{1 6}, 1.3]$ :

$$
\operatorname{spec}_{w} A=\left\{P \in \operatorname{spec} A \mid I_{w} \subseteq P \text { and } P \cap \mathcal{E}_{w}=\varnothing\right\} .
$$

These sets form a disjoint partition of $\operatorname{spec} A[\mathbf{1 5}, 4.1 .1 ; \mathbf{1 6}, 1.2]$.

Fix $w \in \mathcal{W}$, and recall that $I_{w}=Q_{w}$. Note that up to scalars, the products $c_{j, w_{+}}^{+} c_{j, w_{-}}^{-}$coincide with the elements $b_{w, \varpi_{j}}$ (in the notation of $[\mathbf{2 3}, 10.3 .2]$ ). Hence, the cosets $c_{j, w_{+}}^{+} c_{j, w_{-}}^{-}+Q_{w}$ all lie in the Ore set $b_{w}$. Now any $P \in \mathbf{X}(w)$ contains $Q_{w}$, and $P / Q_{w}$ is disjoint from $b_{w}$. Hence, $P \in \operatorname{spec}_{w} A$.

Therefore $\mathbf{X}(w) \subseteq \operatorname{spec}_{w} A$ for all $w \in \mathcal{W}$. Since the $\mathbf{X}(w)$ also form a disjoint partition of $\operatorname{spec} A$, we conclude that $\mathbf{X}(w)=\operatorname{spec}_{w} A$ for all $w \in \mathcal{W}$. 
6.5. For all $i, j \in\{1, \ldots, n\}$ there is a $\mathbb{C}$-algebra automorphism $\tau_{i j}$ of $A$ such that $\tau_{i j}\left(X_{k l}\right)=q^{\delta_{i k}-\delta_{j l}} X_{k l}$ for all $k, l$. For $j \in\{1, \ldots, n\}$ and $y \in S_{n}$, set $\sigma_{j, y}=$ $\prod_{t=1}^{j} \tau_{y(t) t}$. Note that $\sigma_{j, y}\left(X_{k l}\right)=q^{\delta_{k, y J}-\delta_{l, J}} X_{k l}$, where $J=\{1, \ldots, j\}$.

We next observe that the automorphisms $\tau_{i j}$ and $\sigma_{j, y}$ all lie in $\mathcal{H}$. To see this, fix $i$ and $j$, and let $\alpha, \beta \in S L_{n}(\mathbb{C})$ be the diagonal matrices with entries $\alpha_{k k}=q^{\delta_{k i}-(1 / n)}$ and $\beta_{l l}=q^{(1 / n)-\delta_{l j}}$. If $\phi_{\alpha}$ and $\phi_{\beta}$ are the corresponding characters of $A$, then $\theta_{\phi_{\alpha}}^{r} \theta_{\phi_{\beta}}^{\ell}\left(X_{k l}\right)=q^{\delta_{k i}-\delta_{l j}} X_{k l}$ for all $k, l$; thus $\theta_{\phi_{\alpha}}^{r} \theta_{\phi_{\beta}}^{\ell}=\tau_{i j}$. This shows that $\tau_{i j} \in \mathcal{H}$ for all $i, j$, and it follows that $\sigma_{j, y} \in \mathcal{H}$ for all $j, y$.

Lemma. Let $j \in\{1, \ldots, n-1\}, y \in S_{n}, \epsilon= \pm$. Then $c_{j, y}^{\epsilon}$ is $\sigma_{j, y}$-normal modulo the ideal $\left\langle c_{j, z}^{\epsilon} \mid z>_{j} y\right\rangle$. In particular, $c_{j, y}^{\epsilon}$ is $\sigma_{j, y}$-normal modulo $I^{\epsilon}\left(y, \varpi_{j}\right)$.

Proof. Set $J=\{1, \ldots, j\}$. By $[\mathbf{1 6}, 3.1], c_{j, y}^{+} X_{k l}-\sigma_{j, y}\left(X_{k l}\right) c_{j, y}^{+}$lies in the ideal generated by $\left\{c_{j,(a, k) y}^{+} \mid k>a \in y J\right\}$ for all $k, l$. For $k>a \in y J$, note that $(a, k) y J>y J$, so that $(a, k) y>_{j} y$. Therefore $c_{j, y}^{+}$is $\sigma_{j, y}$-normal modulo $\left\langle c_{j, z}^{+}\right|$ $\left.z>_{j} y\right\rangle$.

Now let $D_{k l}=D(\{1, \ldots, n\} \backslash\{k\},\{1, \ldots, n\} \backslash\{l\})$ for all $k, l$, and observe that $\sigma_{j, y}\left(D_{k l}\right)=q^{\left(j-\delta_{k, y J}\right)-\left(j-\delta_{l, J}\right)} D_{k l}$. Hence,

$$
c_{j, y}^{+} D_{k l}-q^{\delta_{l, J}-\delta_{k, y J}} D_{k l} c_{j, y}^{+} \in\left\langle c_{j, z}^{+} \mid z>_{j} y\right\rangle .
$$

Now apply the $\mathbb{C}$-linear antiautomorphism * of $A$ discussed in $[\mathbf{1 5}, 1.2,1.4]$. Since $D_{k l}^{*}=(-q)^{k-l} X_{k l}[\mathbf{1 5}, 1.4 .1(\mathrm{c})]$ and each $\left(c_{j, z}^{+}\right)^{*}$ is a nonzero scalar multiple of $c_{j, z}^{-}$ $[\mathbf{1 5}, 1.6 .1(\mathrm{~b})]$, we see that $\left.X_{k l} c_{j, y}^{-}-q^{\delta_{l, J}-\delta_{k, y J}} c_{j, y}^{-} X_{k l} \in\left\langle c_{j, z}^{-} \mid z\right\rangle_{j} y\right\rangle$ for all $k, l$. Therefore $c_{j, y}^{-}$is $\sigma_{j, y}$-normal modulo $\left\langle c_{j, z}^{-} \mid z>_{j} y\right\rangle$.

6.6. Lemma 6.5 shows that the generators for the ideals $I_{w}=Q_{w}$ given in $(6.3 \mathrm{~d})$ are $\mathcal{H}$-polynormal, and identifies the corresponding automorphisms. The advantage is that we obtain a smaller set of automorphisms with which to describe the links in $\operatorname{spec}_{w} A$ than the set given in (5.9).

Theorem. Let $P$ and $Q$ be distinct primes in some $\operatorname{spec}_{w} A$. If $P \rightsquigarrow Q$, then $P=\sigma_{j, y}(Q)$ for some $j \in\{1, \ldots, n-1\}$ and $y \in S_{n}$ such that either $y \mathbb{Z}_{j} w_{+}$ or $y \overleftrightarrow{\leq}_{j} w_{-}$. Furthermore, $j$ and $y$ can be chosen so that $\sigma_{j, y}(K) \rightsquigarrow K$ for all $K \in \operatorname{spec}_{w} A$ that contain $Q$.

6.7. Let $w \in \mathcal{W}$. By Theorems 3.3 and 6.6, the subgroup $T_{w} \subseteq \mathcal{H}$ determines all the cliques within $\operatorname{spec}_{w} A$, the finite set

$$
\Sigma_{w} \cap\left\{\sigma_{j, y} \mid j=1, \ldots, n-1 ; y \in S_{n} ; y \not \bigsqcup_{j} w_{+} \text {or } y \not \mathbf{Z}_{j} w_{-}\right\}
$$

is a full set of coset representatives for $\Sigma_{w} / N_{w}$, and this set generates $T_{w}$ modulo $N_{w}$. It is easy to see that not all $\sigma_{j, y}$ with $y \not_{j} w_{+}$or $y \not_{j} w_{-}$lie in $\Sigma_{w}$ (cf. Example 6.12); in fact, these $\sigma_{j, y}$ need not even be in $T_{w}$ (Example 6.13). Recall that $T_{w} / N_{w}$ is a free abelian group of rank at most $n-1-s(w)$ (Theorem 5.12). Given the identification of $W$ with $S_{n}$, we can calculate the quantity $s(w)$ as the minimum length of an expression for $w_{-}^{-1} w_{+}$as a product of transpositions.

6.8. In our first example, we give a complete picture of $\operatorname{spec} A$ in the case $n=2$. 
Example $[n=2]$. Observe that the only relation of the form $y \not_{1} z$ in $S_{2}$ is the relation $(1,2) \not \leq_{1} e$, and that $\sigma_{1,(1,2)}=\tau_{21}$. Hence, Theorem 6.6 shows that the only possible links between distinct primes that can occur in spec $A$ are links of the form $\tau_{21}(P) \rightsquigarrow P$. On the other hand, since $X_{21}$ is a regular $\tau_{21}$-normal element of $A$, it follows from Lemma 3.7 that all cliques in spec $A$ are invariant under $\tau_{21}$. Therefore the clique of every prime in spec $A$ coincides with its $\left\langle\tau_{21}\right\rangle$-orbit.

(a) Primes, primitives, and links in $R_{q}\left[S L_{2}(\mathbb{C})\right]$. The following can be confirmed by direct calculation (for the primitive ideals, see [15, B.1.1]):

$$
\begin{aligned}
& \operatorname{spec}_{((1,2),(1,2))} A=\{\langle 0\rangle\} \cup\left\{Q_{\lambda}:=\left\langle X_{21}-\lambda X_{12}\right\rangle \mid \lambda \in \mathbb{C}^{\times}\right\}, \\
& \operatorname{spec}_{((1,2), e)} A=\left\{\left\langle X_{12}\right\rangle\right\}, \\
& \operatorname{spec}_{(e,(1,2))} A=\left\{\left\langle X_{21}\right\rangle\right\}, \\
& \operatorname{spec}_{(e, e)} A=\left\{\left\langle X_{12}, X_{21}\right\rangle\right\} \cup\left\{P_{\lambda}:=\left\langle X_{11}-\lambda, X_{12}, X_{21}, X_{22}-\lambda^{-1}\right\rangle \mid \lambda \in \mathbb{C}^{\times}\right\} .
\end{aligned}
$$

All these primes are primitive except for $\langle 0\rangle$ and $\left\langle X_{12}, X_{21}\right\rangle$. The links among these primes are

$$
P \rightsquigarrow P \quad(0 \neq P \in \operatorname{spec} A) \quad \text { and } \quad P_{q \lambda} \rightsquigarrow P_{\lambda} \quad\left(\lambda \in \mathbb{C}^{\times}\right) .
$$

All the non-maximal primes are AR-ideals and have singleton cliques, whereas all the maximal ideals have infinite cliques (compare [5, 4.3]). The intersection of the clique of any maximal ideal is $\left\langle X_{12}, X_{21}\right\rangle$.

(b) Symplectic leaves in $S L_{2}(\mathbb{C})$. These are listed in [15, B.2.1]; as predicted by the results of that paper, these leaves are in bijection with the primitive ideals listed in (a):

$$
\begin{aligned}
Q_{\lambda} & \longleftrightarrow\left\{\left(\begin{array}{cc}
a & \lambda b \\
b & d
\end{array}\right) \mid a, d \in \mathbb{C} ; b \in \mathbb{C}^{\times} ; a d-\lambda b^{2}=1\right\}, \\
\left\langle X_{12}\right\rangle & \longleftrightarrow\left\{\left(\begin{array}{cc}
a & 0 \\
b & a^{-1}
\end{array}\right) \mid a, b \in \mathbb{C}^{\times}\right\}, \\
\left\langle X_{21}\right\rangle & \longleftrightarrow\left\{\left(\begin{array}{cc}
a & b k \\
0 & a^{-1}
\end{array}\right) \mid a, b \in \mathbb{C}^{\times}\right\}, \\
P_{\lambda} & \longleftrightarrow\left\{\left(\begin{array}{cc}
\lambda & 0 \\
0 & \lambda^{-1}
\end{array}\right)\right\} .
\end{aligned}
$$

(c) Primes, primitives, and links in $U\left(\mathbf{g}_{r}\right)$. Taking the standard presentation of $\mathbf{g}=\mathbf{s l}_{2}(\mathbb{C})$ with basis $e, f, h$, one finds that the Lie algebra $\mathbf{g}_{r}$ of $(0.7)$ has a basis consisting of $x:=(h,-h), y:=(e, 0)$, and $z:=(0, f)$. Then $[y, z]=0,[x, y]=2 y$, $[x, z]=2 z$. One calculates the prime spectrum of $U\left(\mathbf{g}_{r}\right)$ to be

$$
\begin{array}{rrrr}
\langle 0\rangle, & \langle y\rangle, & \langle z\rangle, & \langle y, z\rangle, \\
Q_{\lambda}^{\prime}:=\langle y-\lambda z\rangle & \left(\lambda \in \mathbb{C}^{\times}\right), & P_{\lambda}^{\prime}:=\langle y, z, x-\lambda\rangle & (\lambda \in \mathbb{C}) .
\end{array}
$$

All these primes are primitive except for $\langle 0\rangle$ and $\langle y, z\rangle$. Each of the nonzero prime ideals of $U\left(\mathbf{g}_{r}\right)$ is linked to itself; the only other links are

$$
P_{\lambda+2}^{\prime} \rightsquigarrow P_{\lambda}^{\prime} \quad(\lambda \in \mathbb{C}) .
$$

Therefore the link graph of $\operatorname{spec} U\left(\mathrm{~g}_{r}\right)$ is isomorphic to that of $\operatorname{spec} R_{q}\left[S L_{2}(\mathbb{C})\right]$. 
(d) Symplectic leaves in $\mathbf{g}_{r}^{*}$. As explained in (0.7), these are the orbits in $\mathbf{g}_{r}^{*}$ of the adjoint algebraic group. For this particular example, the calculation appears as [1, Example 1, §12.6] (with their parameter $\alpha=1$; replace our $x$ by $\frac{1}{2} x$ ). Taking $\left\{x^{*}, y^{*}, z^{*}\right\}$ as the dual basis of $\mathbf{g}_{r}^{*}$, one finds that the orbits corresponding to the primitives under the Dixmier map are as follows:

$$
\begin{aligned}
& Q_{\lambda}^{\prime} \longleftrightarrow\left\{a x^{*}+b y^{*}+c z^{*} \mid a, b, c \in \mathbb{C} ; b=\lambda c \neq 0\right\}, \\
& \langle y\rangle \longleftrightarrow\left(\mathbb{C} x^{*}+\mathbb{C} z^{*}\right) \backslash \mathbb{C} x^{*}, \\
& \langle z\rangle \longleftrightarrow\left(\mathbb{C} x^{*}+\mathbb{C} y^{*}\right) \backslash \mathbb{C} x^{*}, \\
& P_{\lambda}^{\prime} \longleftrightarrow\left\{\lambda x^{*}\right\} . \quad \square
\end{aligned}
$$

6.9. To understand fully the cases where $n \geq 3$, it is important to know exactly which quantum minors lie in a given prime ideal. It is, of course, easiest to give a complete answer for the $1 \times 1$ minors, i.e., the generators $X_{i j}$, and we do so below. In the first version of this paper, we conjectured that some analog of this result should hold for $2 \times 2$ and higher quantum minors; such an analog has in the meantime been obtained by Joseph [24, Théorème 2].

Lemma. Let $w \in \mathcal{W}$ and $P \in \operatorname{spec}_{w} A$, and let $i, j \in\{1, \ldots, n\}$. Then $X_{i j} \in P$ if and only if either $i>\max \left\{w_{+}(1), \ldots, w_{+}(j)\right\}$ or $i<\min \left\{w_{-}(j), \ldots, w_{-}(n)\right\}$.

Proof. Assume first that $i>\max \left\{w_{+}(1), \ldots, w_{+}(j)\right\}$. Choose any $x \in S_{n}$ with $x(1)=i$. Then $x>_{1} w_{+}$and so $X_{i 1}=c_{1, x}^{+} \in I_{w} \subseteq P$. This covers the case $j=1$. If $j>1$, we may assume, by induction, that $X_{i 1}, \ldots, X_{i, j-1} \in P$. Choose $y \in S_{n}$ such that $y(k)=w_{+}(k)$ for $k<j$ while $y(j)=i$, and observe that $y \not_{j} w_{+}$. Hence, $c_{j, y}^{+} \in I_{w} \subseteq P$. Expand $c_{j, y}^{+}$along the $i$-th row; since $X_{i 1}, \ldots, X_{i, j-1} \in P$, it follows that

$$
D\left(\left\{w_{+}(1), \ldots, w_{+}(j-1)\right\},\{1, \ldots, j-1\}\right) X_{i j} \in P .
$$

However, $D\left(\left\{w_{+}(1), \ldots, w_{+}(j-1)\right\},\{1, \ldots, j-1\}\right)=c_{j-1, w_{+}}^{+} \in \mathcal{E}_{w}$, and so we conclude that $X_{i j} \in P$.

Assume next that $i<\min \left\{w_{-}(j), \ldots, w_{-}(n)\right\}$. Observe that the permutation $x=\left(i, w_{-}(n)\right) w_{-}$satisfies $x \not_{n-1} w_{-}$, whence $X_{i n}=c_{n-1, x}^{-} \in I_{w} \subseteq P$. This covers the case $j=n$. If $j<n$, we may assume, by induction, that $X_{i, j+1}, \ldots, X_{i n} \in P$. Set $y=\left(i, w_{-}(j)\right) w_{-}$and observe that $y \not_{j-1} w_{-}$. Hence, $c_{j-1, y}^{-} \in I_{w} \subseteq P$. Expanding $c_{j-1, y}^{-}$along the $i$-th row and using the fact that $X_{i, j+1}, \ldots, X_{i n} \in P$, we find that

$$
X_{i j} c_{j, w_{-}}^{-}=X_{i j} D\left(\left\{w_{-}(j+1), \ldots, w_{-}(n)\right\},\{j+1, \ldots, n\}\right) \in P .
$$

Since $c_{j, w_{-}}^{-} \in \mathcal{E}_{w}$, we conclude that $X_{i j} \in P$ in this case also.

For the converse, assume we are given that $X_{i j} \in P$. We claim that one of the following cases must hold:

$$
\begin{array}{ll}
X_{k l} \in P & \text { for all } k \geq i \text { and } l \leq j \\
X_{k l} \in P & \text { for all } k \leq i \text { and } l \geq j .
\end{array}
$$

Consider the expansion of $D$ along the $i$-th row. Since $D=1 \notin P$, we must have $X_{i l_{0}} \notin P$ for some $l_{0}$. Similarly, $X_{k_{0} j} \notin P$ for some $k_{0}$. Suppose first that $l_{0}>j$. 
For any $k>i$, we have $\left(q-q^{-1}\right) X_{i l_{0}} X_{k j}=X_{i j} X_{k l_{0}}-X_{k l_{0}} X_{i j} \in P$ and hence $X_{k j} \in P$, because $X_{i l_{0}} \notin P$. In particular, it follows that $k_{0}<i$. For any $k \geq i$ and $l<j$, we have $\left(q-q^{-1}\right) X_{k_{0} j} X_{k l}=X_{k_{0} l} X_{k j}-X_{k j} X_{k_{0} l} \in P$ and hence $X_{k l} \in P$, because $X_{k_{0} j} \notin P$. Thus, case (SW) holds.

Similarly, if $l_{0}<j$ then case (NE) holds.

In case (SW), we claim that $i>\max \left\{w_{+}(1), \ldots, w_{+}(j)\right\}$. If not, then $i \leq w_{+}(t)$ for some $t \leq j$, and so $X_{w_{+}(t), 1}, \ldots, x_{w_{+}(t), t} \in P$. Considering the expansion of $D$ along the $w_{+}(t)$-th row, we see that $t<n$. On the other hand, by expanding $c_{t, w_{+}}^{+}$along the $w_{+}(t)$-th row, we find that $c_{t, w_{+}}^{+} \in P$, contradicting the fact that $c_{t, w_{+}}^{+} \in \mathcal{E}_{w}$. Therefore $i>\max \left\{w_{+}(1), \ldots, w_{+}(j)\right\}$, as claimed.

An analogous argument shows that $i<\min \left\{w_{-}(j), \ldots, w_{-}(n)\right\}$ in case (NE)

6.10. Corollary. Let $w \in \mathcal{W}$ and $P \in \operatorname{spec}_{w} A$, and let $i, j \in\{1, \ldots, n\}$. If either $i>\max \left\{w_{+}(1), \ldots, w_{+}(j)\right\}$ or $i<\min \left\{w_{-}(j), \ldots, w_{-}(n)\right\}$, then $\tau_{i j}^{ \pm 1}(P) \in$ clique $(P)$.

Proof. We only treat the case that $i>\max \left\{w_{+}(1), \ldots, w_{+}(j)\right\}$; the remaining case is analogous. By Lemma 6.9, we have $X_{k l} \in P$ for all $k \geq i$ and $l \leq j$.

Let $P_{0}$ denote the inverse image of $P$ in the algebra $A_{0}=\mathcal{O}_{q}\left(M_{n}(\mathbb{C})\right)$. We shall keep the notations $X_{i j}$ and $\tau_{i j}$ for the obvious elements and automorphisms of $A_{0}$. Then $X_{k l} \in P_{0}$ for $k \geq i$ and $l \leq j$, and in view of [20,5.3.12 and proof] (or Lemma $2.7)$, it suffices to show that $\tau_{i j}^{ \pm 1}\left(P_{0}\right) \in \operatorname{clique}\left(P_{0}\right)$.

List the ordered pairs $(k, l)$ for $k \geq i$ and $l \leq j$ as a sequence $\left(i_{1}, j_{1}\right),\left(i_{2}, j_{2}\right), \ldots$ in such a way that for $t>s$ either $i_{t} \leq i_{s}$ or $j_{t} \geq j_{s}$. Set $c_{s}=X_{i_{s} j_{s}}$ for all $s$, and observe that each $c_{t}$ is $\tau_{i_{t} j_{t}}$-normal modulo $\left\langle c_{s} \mid s<t\right\rangle$. Also, it is easily checked that the algebra $A_{0} /\left\langle c_{s} \mid s<t\right\rangle$ is an iterated skew polynomial ring starting from $\mathbb{C}$ (adjoin generators $\bar{X}_{k l}$ for $(k, l) \notin\left\{\left(i_{1}, j_{1}\right), \ldots,\left(i_{t-1}, j_{t-1}\right)\right\}$ in lexicographic order). Hence, $A_{0} /\left\langle c_{s} \mid s<t\right\rangle$ is a domain, and so $c_{t}$ is regular modulo $\left\langle c_{s} \mid s<t\right\rangle$. The desired conclusion now follows from Lemma 3.7.

6.11. We can now give an example showing that when $n>2$, no single subgroup of $\mathcal{H}$ can determine all the cliques within $\operatorname{spec} A$.

Example $[n=3]$. Since the transposition $(1,3) \in S_{3}$ is maximal with respect to

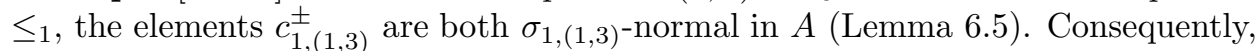
the element $c_{1,(1,3)}^{+}-c_{1,(1,3)}^{-}=X_{31}-X_{12} X_{23}+q X_{13} X_{22}$ is $\sigma_{1,(1,3)}$-normal, and so the ideal $P_{1}=\left\langle X_{31}-X_{12} X_{23}+q X_{13} X_{22}\right\rangle$ is AR. It can be checked that $P_{1}$ is prime. Similarly, the element $c_{2,(1,3)}^{+}-c_{2,(1,3)}^{-}$generates an AR-prime $P_{2}=$ $\left\langle X_{21} X_{32}-q X_{22} X_{31}-X_{13}\right\rangle$. Thus $P_{1}$ and $P_{2}$ have singleton cliques. Let $P_{3}$ denote the maximal ideal

$$
\left\langle X_{11}-1, X_{12}, X_{13}, X_{21}, X_{22}-1, X_{23}, X_{31}, X_{32}, X_{33}-1\right\rangle,
$$

and note that $P_{3} \in \operatorname{spec}_{(e, e)} A$ (cf. Example 6.12). It follows from Corollary 6.10 that $\tau_{21}\left(P_{3}\right) \in$ clique $\left(P_{3}\right)$.

We claim that there is no subgroup $S$ of $\mathcal{H}$ such that the $S$-orbits of $P_{1}, P_{2}$, $P_{3}$ coincide with their cliques. If such a subgroup did exist, there would be some $\tau \in S$ such that $\tau$ fixes $P_{1}, P_{2}$ and $\tau\left(P_{3}\right)=\tau_{21}\left(P_{3}\right)$. Now there exist $\lambda_{i j} \in \mathbb{C}^{\times}$ such that $\tau\left(X_{i j}\right)=\lambda_{i j} X_{i j}$ for all $i, j$. Since $\tau$ fixes $P_{1}$ and $P_{2}$, we must have $\lambda_{31}=\lambda_{12} \lambda_{23}=\lambda_{13} \lambda_{22}$ and $\lambda_{21} \lambda_{32}=\lambda_{22} \lambda_{31}=\lambda_{13}$. Since $\tau\left(P_{3}\right)=\tau_{21}\left(P_{3}\right)$, we 
must have $\lambda_{11}=q^{-1}$ and $\lambda_{22}=q$ while $\lambda_{33}=1$. But then $\lambda_{31}=q \lambda_{13}$ and $\lambda_{13}=q \lambda_{31}$, which is impossible because $\lambda_{31} \neq 0$ and $q^{2} \neq 1$.

Therefore no subgroup of $\mathcal{H}$ determines the cliques of $P_{1}, P_{2}, P_{3}$ simultaneously.

6.12. Our next example exhibits the links and cliques of the maximal ideals of codimension 1.

Example $[w=(e, e)]$. In this case, Lemma 6.9 shows that the primes in $\operatorname{spec}_{w} A$ must contain $X_{i j}$ for $i \neq j$. Conversely, any prime containing those $X_{i j}$ clearly contains $I_{w}$ and avoids $\mathcal{E}_{w}$. Hence, if $J=\left\langle X_{i j} \mid i \neq j\right\rangle$, then

$$
\operatorname{spec}_{w} A=\{P \in \operatorname{spec} A \mid J \subseteq P\} ;
$$

in particular, $\operatorname{spec}_{w} A$ contains all the maximal ideals of codimension 1. By Corollary 6.10, the cliques in $\operatorname{spec}_{w} A$ are invariant under $\tau_{i j}$ for all $i \neq j$. Moreover, since $\tau_{i i}\left(X_{j j}\right)=X_{j j}$ for all $i, j$, each $\tau_{i i}$ induces the identity automorphism on $A / J$; thus $\tau_{i i}$ fixes the primes in $\operatorname{spec}_{w} A$, and hence the cliques in $\operatorname{spec}_{w} A$ are also invariant under $\tau_{i i}$. This shows that $\tau_{i j} \in T_{P}$ for all $i, j$ and all $P \in \operatorname{spec}_{w} A$; in particular, $\tau_{i j} \in T_{w}$ for all $i, j$. Therefore by Theorem 6.6 and Proposition 3.1(c), the group $\left\langle\tau_{i j} \mid i, j=1, \ldots, n\right\rangle$ determines the cliques within $\operatorname{spec}_{w} A$. Alternatively put, the set $\left\{\tau_{i j} \mid i, j=1, \ldots, n\right\}$ generates $T_{w}$ modulo $N_{w}$.

The maximal ideals of $A$ of codimension 1 are the maximal elements of $\operatorname{spec}_{w} A$; these are in bijection with the maximal ideals of the torus

$$
A / J \cong \mathbb{C}\left[x_{11}, \ldots, x_{n n}\right] /\left\langle x_{11} x_{22} \cdots x_{n n}-1\right\rangle,
$$

and so they can be parametrized by the set

$$
\Lambda=\left\{\boldsymbol{\lambda}=\left(\lambda_{1}, \ldots, \lambda_{n}\right) \in \mathbb{C}^{n} \mid \lambda_{1} \lambda_{2} \cdots \lambda_{n}=1\right\} .
$$

Thus $\operatorname{prim}_{w} A=\left\{P_{\boldsymbol{\lambda}} \mid \boldsymbol{\lambda} \in \Lambda\right\}$, where $P_{\boldsymbol{\lambda}}=\left\langle X_{i j}, X_{i i}-\lambda_{i} \mid i, j=1, \ldots, n ; i \neq j\right\rangle$. Since these primes form a single $\mathcal{H}$-orbit, it suffices to consider a single one of them, say $P_{\mathbf{1}}$ where $\mathbf{1}=(1,1, \ldots, 1)$.

Since $A / J$ is a commutative domain, we see directly that $P_{\mathbf{1}} / J \rightsquigarrow P_{1} / J$, and so $P_{\mathbf{1}} \rightsquigarrow P_{\mathbf{1}}$, without appealing to Theorem 2.6. One can exhibit links $\tau_{k+1, k}\left(P_{\mathbf{1}}\right) \rightsquigarrow P_{\mathbf{1}}$ for $k=1, \ldots, n-1$ by direct calculation, but we shall obtain this more easily below. Note that since the primes linked to $P_{\mathbf{1}}$ are in $\operatorname{spec}_{w} A$ and so contain $J$, all links to $P_{\mathbf{1}}$ must arise from links to $P_{\mathbf{1}} / J P_{\mathbf{1}}$ in spec $A / J P_{\mathbf{1}}$.

We next show that $X_{i j} \in J P_{\mathbf{1}}$ whenever $|i-j| \geq 2$. If $j \geq i+2$, then

$$
\left(q-q^{-1}\right) X_{i j} X_{i+1, i+1}=X_{i, i+1} X_{i+1, j}-X_{i+1, j} X_{i, i+1} \in J^{2}
$$

and so $X_{i j} X_{i+1, i+1} \in J^{2}$. Since $X_{i j}\left(X_{i+1, i+1}-1\right) \in J P_{\mathbf{1}}$, it follows that $X_{i j} \in J P_{\mathbf{1}}$. Similarly, if $i \geq j+2$, then

$$
\begin{aligned}
\left(q-q^{-1}\right) X_{i j} X_{i-1, i-1} & =X_{i-1, j} X_{i, i-1}-X_{i, i-1} X_{i-1, j} \in J^{2}, \\
X_{i j}\left(X_{i-1, i-1}-1\right) & \in J P_{\mathbf{1}},
\end{aligned}
$$

and again $X_{i j} \in J P_{\mathbf{1}}$. Thus $J / J P_{\mathbf{1}}$ is generated by the cosets $X_{i j}+J P_{\mathbf{1}}$ where $|i-j|=1$. Observe that for $k=1, \ldots, n-1$, the elements $X_{k+1, k}$ and $X_{k, k+1}$ are 
both $\tau_{k+1, k}$-normal modulo $J P_{\mathbf{1}}$. Since links to $P_{\mathbf{1}}$ all arise from links to $P_{\mathbf{1}} / J P_{\mathbf{1}}$, Lemma 2.7 now shows that the only primes that can possibly be linked to $P_{\mathbf{1}}$ are $P_{\mathbf{1}}, \tau_{21}\left(P_{\mathbf{1}}\right), \tau_{32}\left(P_{\mathbf{1}}\right), \ldots, \tau_{n, n-1}\left(P_{\mathbf{1}}\right)$.

In the notation of (3.3), we now have that $\left\{\mathrm{id}, \tau_{21}, \ldots, \tau_{n, n-1}\right\}$ contains a transversal for $\Sigma_{w} / N_{w}$; in particular, $\tau_{21}, \ldots, \tau_{n, n-1}$ generate $T_{w}$ modulo $N_{w}$. Since any monomial of the form $\tau_{21}^{m_{1}} \tau_{32}^{m_{2}} \cdots \tau_{n, n-1}^{m_{n-1}}$ maps $P_{\mathbf{1}}$ to $P_{\boldsymbol{\lambda}}$, where

$$
\boldsymbol{\lambda}=\left(q, q^{-1}, 1, \ldots, 1\right)^{m_{1}}\left(1, q, q^{-1}, 1, \ldots, 1\right)^{m_{2}} \cdots\left(1, \ldots, 1, q, q^{-1}\right)^{m_{n-1}},
$$

we see that $\tau_{21}^{m_{1}} \tau_{32}^{m_{2}} \cdots \tau_{n, n-1}^{m_{n-1}} \in N_{w}$ only when $m_{1}=m_{2}=\cdots=m_{n-1}=0$. Therefore $T_{w} / N_{w}$ is a lattice of rank $n-1$. Consequently, no proper subset of $\left\{\tau_{21}, \ldots, \tau_{n, n-1}\right\}$ is large enough to generate $T_{w} / N_{w}$, and so no proper subset of $\left\{\right.$ id, $\left.\tau_{21}, \ldots, \tau_{n, n-1}\right\}$ can be a transversal for $\Sigma_{w} / N_{w}$. (Recall from Proposition $3.1(\mathrm{~d})$ that $\Sigma_{w}$ generates $T_{w}$.) Thus $\left\{\mathrm{id}, \tau_{21}, \ldots, \tau_{n, n-1}\right\}$ must be contained in $\Sigma_{w}$ and

$$
\Sigma_{w}=N_{w} \cup \tau_{21} N_{w} \cup \tau_{32} N_{w} \cup \cdots \cup \tau_{n, n-1} N_{w} .
$$

Therefore we conclude from Theorem 3.3(b) that

$$
\left\{Q \in \operatorname{spec}_{w} A \mid Q \rightsquigarrow P\right\}=\left\{P, \tau_{21}(P), \ldots, \tau_{n, n-1}(P)\right\}
$$

for all $P \in \operatorname{spec}_{w} A$.

6.13. In our final example, we show that the automorphisms $\sigma_{j, y}$ appearing in Theorem 6.6 do not always correspond to the clique of the prime in question.

Example $[n=4]$. Let $w_{+}=(1,3,4)$ and $w_{-}=(1,4)(2,3)$. Then $y \leq_{j} w_{-}$for all $y \in S_{4}$ and $j \leq 3$. On the other hand, the non-inequalities $y \not_{j} w_{+}$have the following solutions:

$$
\begin{array}{ll}
j=1: & y(1)=4 ; \\
j=2: & y(\{1,2\})=\{1,4\},\{2,4\},\{3,4\} \\
j=3: & \text { no solutions. }
\end{array}
$$

The corresponding automorphisms $\sigma_{j, y}$ may be listed as follows:

$$
\left\{\sigma_{j, y} \mid y \not_{j} w_{+}\right\}=\left\{\tau_{41}, \tau_{11} \tau_{42}, \tau_{21} \tau_{42}, \tau_{31} \tau_{42}\right\} .
$$

(The automorphisms $\sigma_{2, y}$ depend only on the sets $y(\{1,2\})$; for instance, $\tau_{11} \tau_{42}=$ $\left.\tau_{41} \tau_{12}.\right)$ Thus, by Theorem 6.6 , nontrivial links in $\operatorname{spec}_{w} A$ can only be given by the above four automorphisms, i.e., in the notation of Section 3, those of $\tau_{41}, \tau_{11} \tau_{42}, \tau_{21} \tau_{42}, \tau_{31} \tau_{42}$ which are in $\Sigma_{w}$ generate $T_{w}$ modulo $N_{w}$. We shall see that, in fact, $\tau_{11} \tau_{42} \notin T_{w}$.

Now $I_{w_{-}}^{-}=0$, and $I_{w_{+}}^{+}$is generated by the elements

$$
X_{41}, X_{11} X_{42}-q X_{12} X_{41}, X_{21} X_{42}-q X_{22} X_{41}, X_{31} X_{42}-q X_{32} X_{41} ;
$$

therefore $I_{w}=\left\langle X_{41}, X_{11} X_{42}, X_{21} X_{42}, X_{31} X_{42}\right\rangle$. Since also $X_{11}, X_{21}, X_{31}, X_{41}$ generate $A$ as a left ideal, we see that in fact $I_{w}=\left\langle X_{41}, X_{42}\right\rangle$. Now $X_{41}$ is $\tau_{41}$ normal in $A$, and $X_{42}$ is $\tau_{42}$-normal modulo $\left\langle X_{41}\right\rangle$. Hence, Lemma 2.7 shows that 
nontrivial links in $\operatorname{spec}_{w} A$ can only be given by $\tau_{41}$ or $\tau_{42}$. Thus, $\Sigma_{w} \cap\left\{\tau_{41}, \tau_{42}\right\}$ generates $T_{w}$ modulo $N_{w}$. Further, $\tau_{41}, \tau_{42} \in T_{w}$ by Corollary 6.10 , and therefore $\tau_{41}$ and $\tau_{42}$ generate $T_{w}$ modulo $N_{w}$.

We claim that $T_{w}=N_{w}$, which we can see with the help of Lemma 5.11 once $Z_{w}$ is identified. Recall $[\mathbf{2 3}, 10.3 .3]$ that $Z_{w}=\mathbb{C}\left[a_{w, \mu_{1}}^{ \pm}, \ldots, a_{w, \mu_{r}}^{ \pm}\right]$, where $\mu_{1}, \ldots$, $\mu_{r}$ is a basis for the lattice

$$
P_{w}(\pi)=\left\{\lambda \in P(\pi) \mid w_{+} \lambda=w_{-} \lambda\right\} .
$$

For the current example, we compute that

$$
\begin{array}{ll}
w_{+} \varpi_{1}=-\varpi_{2}+\varpi_{3}, & w_{-} \varpi_{1}=-\varpi_{3}, \\
w_{+} \varpi_{2}=-\varpi_{1}+\varpi_{3}, & w_{-} \varpi_{2}=-\varpi_{2}, \\
w_{+} \varpi_{3}=-\varpi_{1}, & w_{-} \varpi_{3}=-\varpi_{1} .
\end{array}
$$

It follows that $P_{w}(\pi)=\mathbb{Z} \varpi_{3}$, and hence $Z_{w}=\mathbb{C}\left[a_{w, \varpi_{3}}^{ \pm 1}\right]$. Since

$$
\begin{aligned}
a_{w, \varpi_{3}} & =\left(c_{w_{+} \varpi_{3}, \varpi_{3}}^{\varpi_{3}}\right)^{-1} c_{w_{-} w_{0} \varpi_{1}, w_{0} \varpi_{1}}^{\varpi_{1}} \\
& =\left(c_{3, w_{+}}^{+}\right)^{-1} c_{3, w_{-}}^{-}=D(\{2,3,4\},\{1,2,3\})^{-1} X_{14},
\end{aligned}
$$

we see that $\tau_{41}\left(a_{w, \varpi_{3}}\right)=\tau_{42}\left(a_{w, \varpi_{3}}\right)=a_{w, \varpi_{3}}$. Hence, $\tau_{41}$ and $\tau_{42}$ restrict to the identity automorphism of $Z_{w}$, and thus $\tau_{41}, \tau_{42} \in N_{w}$ by Lemma 5.11. Since $\tau_{41}$ and $\tau_{42}$ generate $T_{w}$ modulo $N_{w}$, it follows that $T_{w}=N_{w}$. In particular, all cliques in $\operatorname{spec}_{w} A$ are trivial.

Finally, observe that $\tau_{11} \tau_{42}\left(a_{w, \varpi_{3}}\right)=q^{2} a_{w, \varpi_{3}}$, whence $\tau_{11} \tau_{42}$ does not restrict to the identity on $Z_{w}$. Since $T_{w}=N_{w}$, we therefore conclude from Lemma 5.11 that $\tau_{11} \tau_{42} \notin T_{w}$. This shows that the automorphisms $\sigma_{j, y}$ with $y \not_{j} w_{+}$or $y \not_{j} w_{-}$do not always lie in $T_{w}$.

The main point of the example above is that the "obvious" conjecture for a set of generators for $T_{w} / N_{w}$ (based on Theorem 6.6) is false. The problem of giving an explicit description of the groups $T_{w} / N_{w}$ remains open, as does the question of determining the sets $\Sigma_{w} / N_{w}$.

6.14. The quantized function algebra $R_{q}\left[G L_{n}(\mathbb{C})\right]$ is given by the same generators and relations as $R_{q}\left[S L_{n}(\mathbb{C})\right]$ (see (6.1)) except that $\operatorname{Det}_{q} X$ is inverted rather than being set equal to 1 . Corresponding to the natural embedding $G L_{n}(\mathbb{C}) \hookrightarrow$ $S L_{n+1}(\mathbb{C})$ given by the rule

$$
x \mapsto\left(\begin{array}{cc}
x & 0 \\
0 & (\operatorname{det} x)^{-1}
\end{array}\right),
$$

there is a surjective $\mathbb{C}$-algebra homomorphism $\mathcal{O}\left(S L_{n+1}(\mathbb{C})\right) \rightarrow \mathcal{O}\left(G L_{n}(\mathbb{C})\right)$. Inspection of the relations for $R_{q}\left[S L_{n+1}(\mathbb{C})\right]$ and $R_{q}\left[G L_{n}(\mathbb{C})\right]$ reveals that we have, analogously,

$$
R_{q}\left[S L_{n+1}(\mathbb{C})\right] /\left\langle X_{n+1,1}, \ldots, X_{n+1, n}, X_{1, n+1}, \ldots, X_{n, n+1}\right\rangle \cong R_{q}\left[G L_{n}(\mathbb{C})\right] .
$$

In particular, it follows immediately from Theorem 5.8 that $\operatorname{spec} R_{q}\left[G L_{n}(\mathbb{C})\right]$ has normal separation and that $R_{q}\left[G L_{n}(\mathbb{C})\right]$ satisfies the strong second layer condition. Similarly, the other results of Section 5 yield, after specialization to $R_{q}\left[S L_{n+1}(\mathbb{C})\right]$ and passage to the quotient, corresponding results for $R_{q}\left[G L_{n}(\mathbb{C})\right]$. It is also possible to transfer our results from $R_{q}\left[S L_{n}(\mathbb{C})\right]$ to $R_{q}\left[G L_{n}(\mathbb{C})\right]$ by means of the isomorphism $R_{q}\left[G L_{n}(\mathbb{C})\right] \cong R_{q}\left[S L_{n}(\mathbb{C})\right]\left[z, z^{-1}\right]$ given in $[\mathbf{2 8}]$. We leave the details to the interested reader. 


\section{ACKNOWLEDGMENT}

We thank T. Levasseur for helpful comments, and the referee for extensive suggestions.

\section{REFERENCES}

1. W. Borho, P. Gabriel, and R. Rentschler, Primideale in Einhüllenden auflösbarer LieAlgebren, Lecture Notes in Math. 357, Springer-Verlag, Berlin, 1973. MR 51:12965

2. K. A. Brown, On the representation theory of solvable Lie algebras II: the abelian group attached to a prime ideal, J. London Math. Soc. (2) 43 (1991), 49-60. MR 92g:17010

3. K. A. Brown and F. du Cloux, On the representation theory of solvable Lie algebras, Proc. London Math. Soc. (3) 57 (1988), 284-300. MR 89k:17026

4. K. A. Brown and R. B. Warfield, Jr., The influence of ideal structure on representation theory, J. Algebra 116 (1988), 294-315. MR 89k:16026

5. W. Chin and I. M. Musson, Hopf algebra duality, injective modules, and quantum groups, Communic. in Algebra 22 (1994), 4661-4692. MR 95d:16049

6. C. De Concini and C. Procesi, Quantum groups, in D-Modules, Representation Theory, and Quantum Groups (Venezia, June 1992) (L. Boutet de Monvel, G. Zampieri, and A. D'Agnolo, eds.), Lecture Notes in Math. 1565, Springer-Verlag, Berlin, 1993, pp. 31-140. MR 95j: 17012

7. J. Dixmier, Enveloping Algebras, North-Holland, Amsterdam, 1977. MR 58:16803b

8. V. G. Drinfel'd, Quantum groups, in Proc. Internat. Congr. Mathematicians, Berkeley 1986, I, pp. 798-820. MR 89f: 17017

9. A. W. Goldie and G. O. Michler, Ore extensions and polycyclic group rings, J. London Math. Soc. (2) 9 (1974), 337-345. MR 50:9968

10. K. R. Goodearl, Classical localizability in solvable enveloping algebras and PoincaréBirkhoff-Witt extensions, J. Algebra 132 (1990), 243-262. MR 91j:16021

11. K. R. Goodearl and T. H. Lenagan, Catenarity in quantum algebras, J. Pure Appl. Algebra (to appear).

12. K. R. Goodearl and E. S. Letzter, Prime factor algebras of the coordinate ring of quantum matrices, Proc. Amer. Math. Soc. 121 (1994), 1017-1025. MR 94j:16066

13. K. R. Goodearl and R. B. Warfield, Jr., An Introduction to Noncommutative Noetherian Rings, London Math. Soc. Student Text Series 16, Cambridge Univ. Press, Cambridge, 1989. MR 91c: 16001

14. V. Guillemin and S. Sternberg, Geometric Asymptotics, Math. Surveys 14, Amer. Math. Soc., Providence, 1977. MR 58:24404

15. T. J. Hodges and T. Levasseur, Primitive ideals of $\mathbf{C}_{q}[S L(3)]$, Comm. Math. Phys. 156 (1993), 581-605. MR 94k:17023

16. , Primitive ideals of $\mathbf{C}_{q}[S L(n)]$, J. Algebra 168 (1994), 455-468. MR 95i:16038

17. T. J. Hodges, T. Levasseur, and M. Toro, Algebraic structure of multi-parameter quantum groups, Advances in Math. (to appear).

18. J. E. Humphreys, Reflection Groups and Coxeter Groups, Cambridge Univ. Press, Cambridge, 1990. MR 92h:20002

19. N. Jacobson, Structure of Rings, Colloq. Publ. 37, Amer. Math. Soc., Providence, 1956. MR 18:373d

20. A. V. Jategaonkar, Localization in Noetherian Rings, London Math. Soc. Lecture Note Series 98, Cambridge Univ. Press, Cambridge, 1986. MR 88c:16005

21. A. Joseph, Idéaux premiers et primitifs de l'algèbre des fonctions sur un groupe quantique, C. R. Acad. Sci. Paris, Sér. I 316 (1993), 1139-1142. MR 94c:17029

22. _ On the prime and primitive spectra of the algebra of functions on a quantum group, J. Algebra 169 (1994), 441-511. CMP 95:2

23. —_ Quantum Groups and Their Primitive Ideals, Ergeb. der Math. und ihrer Grenzgeb. (3) 29, Springer-Verlag, Berlin, 1995. CMP 95:7

24. Sur les ideaux génériques de l'algèbre des fonctions sur un groupe quantique, C. R. Acad. Sci. Paris, Sér. I 321 (1995), 135-140.

25. A. Joseph and G. Letzter, Separation of variables for quantized enveloping algebras, Amer. J. Math. 116 (1994), 127-177. MR 95e:17017 
26. A. A. Kirillov, Elements of the Theory of Representations, Springer-Verlag, Berlin, 1976. MR 54:447

27. T. H. Lenagan and E. S. Letzter, The fundamental prime ideals of a noetherian prime PI ring, Proc. Edinburgh Math. Soc. 33 (1990), 113-121. MR 91b:16026

28. T. Levasseur and J. T. Stafford, The quantum coordinate ring of the special linear group, J. Pure Appl. Algebra 86 (1993), 181-186. MR 94d:16038

29. J.-H. Lu and A. Weinstein, Poisson Lie groups, dressing transformations and Bruhat decompositions, J. Diff. Geom. 31 (1990), 501-526. MR 91c:22012

30. J. C. McConnell and J. J. Pettit, Crossed products and multiplicative analogues of Weyl algebras, J. London Math. Soc. (2) 38 (1988), 47-55. MR 90e:16011

31. J. C. McConnell and J. C. Robson, Noncommutative Noetherian Rings, Wiley-Interscience, New York, 1987. MR 89j:16023

32. I. M. Musson, Links between cofinite prime ideals in quantum function algebras, Preprint (1995).

33. M. Noumi, H. Yamada, and K. Mimachi, Finite dimensional representations of the quantum group $G L_{q}(n ; \mathbb{C})$ and the zonal spherical functions on $U_{q}(n-1) \backslash U_{q}(n)$, Japanese J. Math. 19 (1993), 31-80. MR 94i:17023

34. B. Parshall and J.-P. Wang, Quantum linear groups, Memoirs Amer. Math. Soc. 89 (1991), no. 439. MR 91g:16028

35. D. S. Passman, Infinite Crossed Products, Academic Press, New York, 1989. MR 90g: 16002

36. M. A. Semenov-Tian-Shansky, What is a classical r-matrix?, Func. Anal. Applic. 17 (1983), 259-272. MR 85i:58061

37. S. P. Smith, Quantum groups: An introduction and survey for ring theorists, in Noncommutative Rings (S. Montgomery and L. Small, eds.), M.S.R.I. Publ. 24, Springer-Verlag, New York, 1992, pp. 131-178. MR 94g:17032

38. Ya. S. Soibelman, The algebra of functions on a compact quantum group, and its representations, Leningrad Math. J. 2 (1991), 161-178. MR 91i:58053a,b (Russian original)

39. R. B. Warfield, Jr., Review of "Localization in Noetherian Rings" by A. V. Jategaonkar, Bull. Amer. Math. Soc. 17 (1987), 396-400.

Department of Mathematics, University of California, Santa Barbara, California 93106

E-mail address: goodearl@math.ucsb.edu

Department of Mathematics, University of Glasgow, Glasgow G12 8QW, Scotland

E-mail address: kab@maths.gla.ac.uk 